\title{
Syntheses and Reactivity of Heterometallic Oxo-Acetylide Cluster Compounds. Skeletal Rearrangement and Conversion of Acetylide to Alkenyl, Alkylidene, and Allenyl Ligands on a WRe, Framework
}

\author{
Yun Chi, ${ }^{*},{ }^{\dagger}$ Hsiao-Ling Wu, ${ }^{\dagger}$ Chi-Chung Chen, ${ }^{\dagger}$ Chi-J ung Su, ${ }^{\dagger}$ \\ Shie-Ming Peng, ${ }^{*}, \neq$ and Gene-Hsiang Lee \\ Department of Chemistry, National Tsing Hua University, Hsinchu 30043, and Department of \\ Chemistry and Instrumentation Center, National Taiwan University, \\ Taipe 10764, Taiwan, Republic of China
}

Received November 5, $1996^{\otimes}$

\begin{abstract}
Oxidation of the mixed-metal cluster $\mathrm{Cp}^{*} \mathrm{WRe}_{2}(\mathrm{CCR})(\mathrm{CO})_{9}\left(\mathbf{1}, \mathrm{Cp}^{*}=\mathrm{C}_{5} \mathrm{Me}_{5} ; \mathrm{R}=\mathrm{Ph}\right.$ and $\mathrm{C}(\mathrm{Me})=\mathrm{CH}_{2}$ ) with dioxygen in solution at $100^{\circ} \mathrm{C}$ affords the oxo clusters $\mathrm{Cp} * \mathrm{~W}(\mathrm{O}) \mathrm{Re}_{2}(\mathrm{CCR})$ $(\mathrm{CO})_{8},\left(\mathbf{2 a}, \mathrm{R}=\mathrm{Ph}\right.$; and $\mathbf{2 b}, \mathrm{R}=\mathrm{C}(\mathrm{Me})=\mathrm{CH}_{2}$. Treatment of $\mathbf{2}$ with $\mathrm{CO}$ at $110{ }^{\circ} \mathrm{C}$ provides the clusters $\mathrm{Cp} * \mathrm{~W}(\mathrm{O}) \mathrm{Re}_{2}(\mathrm{CCR})(\mathrm{CO})_{9}(\mathbf{4})$, which revert back to $\mathbf{2}$ by removal of one CO upon thermolysis. Both compounds $\mathbf{2}$ and $\mathbf{4}$ contain an open triangular skeletal arrangement, a multisite bound acetylide ligand, and a terminal oxoligand attached to the $\mathrm{W}$ atom. Complex $2 \mathbf{a}$ reacts with dihydrogen to form a mixture of three cluster complexes: the acetylide cluster $\mathrm{Cp} * \mathrm{WRe}_{2}(\mu-\mathrm{O})(\mu-\mathrm{H})_{2}(\mathrm{CCPh})(\mathrm{CO})_{6}(5 \mathrm{a})$, alkenyl cluster $\mathrm{Cp} * \mathrm{~W}(\mathrm{O}) \mathrm{Re}_{2}(\mathrm{CHCHPh})(\mathrm{CO})_{8}(\mathbf{6 a})$, and the alkylidene cluster $\mathrm{Cp} * \mathrm{~W}(\mathrm{O}) \mathrm{Re}_{2}(\mu-\mathrm{H})\left(\mathrm{CHCH}_{2} \mathrm{Ph}\right)(\mathrm{CO})_{8}(\mathbf{7 a})$, which are formally produced by addition of two $\mathrm{H}_{2}$ and elimination of two $\mathrm{CO}$ molecules, transferring one $\mathrm{H}_{2}$ to the acetylide ligand and incorporation of one $\mathrm{H}_{2}$ molecule to $\mathbf{6 a}$, respectively. For the vinylacetylide compound $\mathbf{2} \mathbf{b}$, it reacts with dihydrogen under similar conditions to furnish a mixture of the above mentioned clusters $\mathbf{5 b} \mathbf{b} \mathbf{6 b}$, and $\mathbf{7 b}$, together with a fourth allenyl cluster $\mathrm{Cp}^{*} \mathrm{WRe}_{2}(\mu-\mathrm{O})\left(\mathrm{CHCCMe}_{2}\right)(\mathrm{CO})_{7}(\mathbf{8})$, which is probably produced through a $1,3-\mathrm{H}$ migration on the alkenyl ligand $\mathrm{CH}=\mathrm{CHC}(\mathrm{Me})=\mathrm{CH}_{2}$ in $\mathbf{6 b}$. The X-ray structural analysis of these oxo cluster compounds, their spectroscopic data, and the mechanistic studies of the conversion from acetylide clusters $\mathbf{2}, \mathbf{4}$, and $\mathbf{5}$ to alkenyl clusters $\mathbf{6}$, alkylidene clusters $\mathbf{7}$, and allenyl cluster $\mathbf{8}$ are presented.
\end{abstract}

\section{Introduction}

Organometallic complexes containing oxo ligands serve as realistic models for metal-mediated oxidation and other homogeneous and heterogeneous reactions with high-valent metal species as catalysts. ${ }^{1}$ As a result, a large number of oxo metal complexes have been synthesized; their reactivities with the organic substrates have been thoroughly explored. ${ }^{2}$ The critical information obtained has hel ped chemists to understand the basic reaction pattern of the oxygen atom transfer reactions using discrete oxo complexes as catalysts. ${ }^{3}$ Another exploration is to synthesize complexes containing both oxo and hydrocarbyl ligands and to examine their chemistry, with the goal of probing the oxoligand in promoting the various transformations on metal

\footnotetext{
† National Tsing Hua University.
}

₹ National Taiwan University.

\& Abstract published in Advance ACS Abstracts, May 1, 1997.

(1) (a) Nugent, W. A.; Mayer, J . M. Metal-Ligand Multiple Bonds; Wiley-Interscience: New York, 1988. (b) Atagi, L. M.; Over, D. E.; McAlister, D.R.; Mayer, J. M.J . Am. Chem. Soc. 1991, 113, 870. (c) Dobbs, D. A.; Bergman, R. G. J . Am. Chem. Soc. 1993, 115, 3836. (d) Legzdins, P.; Phillips, E. C.; Rettig, S. J .; Trotter, J .; Veltheer, J . E.; Yee, V. C. Organometallics 1992, 11, 3104. (e) Rau, M. S.; Kertz, C. M.; Mercando, L. A.; Geoffroy, G. L.; Rheingold, A. L. J . Am. Chem. Soc. 1991, 113, 7420. (f) Schauer, C. K.; Voss, E.J .; Sabat, M.; Shriver, D. F. J . Am. Chem. Soc. 1989, 111, 7662.

(2) (a) Holm, R. H. Chem. Rev. 1987, 87, 1401. (b) Holm, R. H.; Donahue, J. P. Polyhedron 1993, 12, 571. (c) Woo, L. K. Chem. Rev. 1993, 93, 1125. (d) Dickman, M. H.; Pope, M. T. Chem. Rev. 1994, 94, 569. complexes. The investigation of the reactivities of complexes $\mathrm{MeReO}_{3}, \mathrm{Re}(\mathrm{O}) \mathrm{H}\left(\mathrm{C}_{2} \mathrm{Et}_{2}\right)_{2}$, and $\left(\mathrm{HB}(\mathrm{pz})_{3}\right) \mathrm{ReO}-$ $(\mathrm{Ph}) \mathrm{Cl}$ serve as typical examples of such an approach. ${ }^{4}$

With a goal of pursuing this research objective, we have first prepared a series of trinuclear mixed-metal acetylide cluster compounds with the empirical formula $\mathrm{Cp} * \mathrm{WRe}_{2}(\mathrm{CCR})(\mathrm{CO})_{9}(\mathbf{1})$, where $\mathrm{R}=\mathrm{Ph}$, cyclohexenyl, and $\mathrm{C}(\mathrm{Me})=\mathrm{CH}_{2}$, and examined the chemical reactivity with $\mathrm{H}_{2}$, alcohol, and thiophenol. ${ }^{5}$ Then, complexes 1 were subjected to oxidation by exposure to oxygen gas, which led to the successful isolation of oxo-containing acetylide cluster compounds $\mathrm{Cp} * \mathrm{~W}(\mathrm{O}) \mathrm{Re}_{2}(\mathrm{CCR})(\mathrm{CO})_{8}$ (2), $\mathrm{R}=\mathrm{Ph}$ and $\mathrm{C}(\mathrm{Me})=\mathrm{CH}_{2}$, in high yield. ${ }^{6}$ The oxo complexes $\mathbf{2}$ have been shown to undergo fragmentation upon treatment with thiophenol in toluene to afford a

(3) (a) Clarke, R.; Gahagan, M.; Mackie, R. K.; Foster, D. F.; ColeHamilton, D. J .; Nicol, M.; Montford, A. W. J . Chem. Soc., Dalton Trans. 1995, 1221. (b) Herrmann, W. A.; Fischer, R. W.; Rauch, M. U.; Scherer, W. J . Mol. Catal. 1994, 86, 243. (c) Herrmann, W. A. J . Organomet. Chem. 1995, 500, 149. (d) Herrmann, W. A.; Correia, J. D. G.; Kuehn, F. E.; Artus, G. R. J .; Romao, C. C. Chem. Eur. J . 1996, 2, 168. (e) Belgacem, J .; Kress, J .; Osborn, J . A. J . Mol. Catal. 1994, $86,267$.

(4) (a) Brown, S. N.; Mayer, J . M. Organometallics 1995, 14, 2951. (b) Herrmann, W. A.; Roesky, P. W.; Wang, M.; Scherer, W. Organometallics 1994, 13, 4531. (c) Cundari, T. R.; Conry, R. R.; Spaltenstein, E.; Critchlow, S. C.; Hall, K. A.; Tahmassebi, S. K.; Mayer, J. M. Organometallics 1994, 13, 322. (d) Tahmassebi, S. K.; Conry, R. R.; Mayer, J. M. J . Am. Chem. Soc. 1993, 115, 7553.

(5) Peng, J .-J .; Horng, K.-M.; Cheng, P.-S.; Chi, Y.; Peng, S.-M.; Lee, G.-H. Organometallics 1994, 13, 2365.

(6) Chi, Y.; Cheng, P.-S.; Wu, H.-L.; Hwang, D.-K.; Peng, S.-M.; Lee, G.-H. J . Chem. Soc., Chem Commun. 1994, 1839. 
Scheme 1

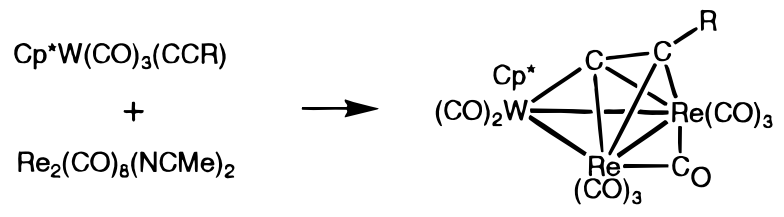

(1)

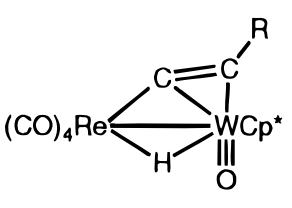

(3)

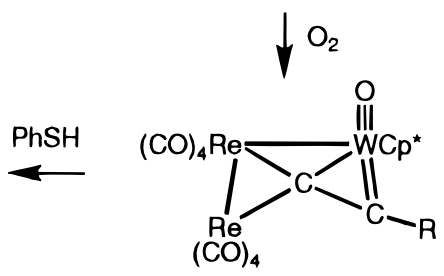

(2) $\mathrm{R}=\mathrm{Ph}, \mathrm{C}(\mathrm{Me})=\mathrm{CH}_{2}$ dinuclear complex Cp*W(O)Re $(\mu-\mathrm{H})(\mathrm{CCR})(\mathrm{CO})_{4} \quad$ (3) (Scheme 1), ${ }^{7}$ while the corresponding reactions of 1 with the same reagent only afforded the addition products, giving cluster compounds $\mathrm{Cp}^{*} \mathrm{WRe}_{2}\left(\mu_{3}-\mathrm{SPh}\right)(\mathrm{CH}=\mathrm{CPh})$ $(\mathrm{CO})_{8}$ and $\mathrm{Cp} * \mathrm{WRe} 2\left(\mu_{3}-\mathrm{SPh}\right)(\mathrm{CH}=\mathrm{CPh})(\mathrm{CO})_{7}$ as the final products. ${ }^{8}$ Such divergence in reactivity between $\mathbf{1}$ and $\mathbf{2}$ has provided the preliminary evidence about the potential influence of the oxo ligand on the chemical reactivities. In this study, we report the experimental results on the X-ray structural analyses and the reactivities of the W-Re oxo-acetylide complexes $\mathbf{2}$ toward $\mathrm{CO}$ and $\mathrm{H}_{2}$, which offer an opportunity for further investigating this phenomenon.

\section{Experimental Procedures}

General Information and Materials. Infrared spectra were recorded on a Perkin Elmer 2000 FT-IR spectrometer. ${ }^{1} \mathrm{H}$ and ${ }^{13} \mathrm{C}$ NMR spectra were recorded on a Bruker AM-400, a Varian Gemini-300 or a Varian Unity-400 instrument. Chemical shifts are quoted with respect to the internal standard tetramethylsilane $\left({ }^{1} \mathrm{H}\right.$ and ${ }^{13} \mathrm{C}$ NMR). Mass spectra were obtained on a J E OL-HX110 instrument operating in fast atom bombardment (FAB) mode. All reactions were performed under a nitrogen atmosphere using deoxygenated solvents dried with an appropriate reagent. Reactions were monitored by analytical thin-layer chromatography ( $5735 \mathrm{Kiesel}$ gel 60 $\mathrm{F}_{254}, \mathrm{E}$. Merck), and the products were separated on commercially available preparative TLC plates (Kieselgel $60 \mathrm{~F}_{254}$, E. Merck). The metal acetylides $\mathrm{Cp} * \mathrm{~W}(\mathrm{CO})_{3}(\mathrm{CCR}), \mathrm{R}=\mathrm{Ph}$ and $\mathrm{C}(\mathrm{Me})=\mathrm{CH}_{2}$, and the rhenium acetonitrile complex $\mathrm{Re}_{2}-$ $(\mathrm{CO})_{8}(\mathrm{NCMe})_{2}$ were prepared according to literature procedures. ${ }^{9}$ The acetylide clusters $\mathrm{Cp} * \mathrm{WRe}_{2}(\mathrm{CCR})(\mathrm{CO})_{9}(\mathbf{1})$ were obtained from the reaction of $\mathrm{Cp} * \mathrm{~W}(\mathrm{CO})_{3}(\mathrm{CCR})$ with $\mathrm{Re}_{2}(\mathrm{CO})_{8^{-}}$ $(\mathrm{NCMe})_{2}$ in toluene. Elemental analyses were performed at the NSC Regional Instrumentation Center at National Cheng Kung University, Tainan, Taiwan.

Synthesis of $\mathbf{C p} * \mathbf{W}(\mathbf{O}) \mathbf{R e}_{2}(\mathbf{C C P h})(\mathbf{C O})_{8}$. To a $100 \mathrm{~mL}$ round-bottomed flask, $\mathrm{Cp} * \mathrm{WRe} \mathrm{e}_{2}(\mathrm{CCPh})(\mathrm{CO})_{9}(\mathbf{l a}, 352 \mathrm{mg}$, $0.337 \mathrm{mmol}$ ) was dissolved into a mixture of toluene $(30 \mathrm{~mL})$ and $\mathrm{n}$-hexane $(30 \mathrm{~mL})$. The solution was then bought to reflux under oxygen, during which the temperature of the oil bath was kept at $100^{\circ} \mathrm{C}$ to avoid over heating the reaction mixture. After $2.5 \mathrm{~h}$, the col or of the solution changed from orange-red to dark-red. The reaction was stopped, and the solvent was

(7) Wu, H.-L.; Lu, G.-L.; Chi, Y.; Farrugia, L. J .; Peng, S.-M.; Lee, G.-H. Inorg. Chem. 1996, 35, 6015.

(8) Peng, J.-J .; Peng, S.-M.; Lee, G.-H.; Chi, Y. Organometallics 1995, $14,626$.

(9) (a) Bruce, M. I.; Humphrey, M. G.; Matisons, J . G.; Roy, S. K.; Swincer, A. G. Aust. J. Chem. 1984, 37, 1955. (b) Harris, G. W.; Boeyens, J. C. A.; Coville, N. J . J . Chem. Soc., Dalton Trans. 1985, 2277. evaporated. The residue was taken in $\mathrm{CH}_{2} \mathrm{Cl}_{2}$, separated by thin-layer chromatography (silica gel, dichloromethane:hexane $=1: 1$ ), and recrystallized from dichloromethane-methanol, giving $191 \mathrm{mg}$ of $\mathrm{Cp} * \mathrm{~W}(\mathrm{O}) \mathrm{Re}_{2}(\mathrm{CCPh})(\mathrm{CO})_{8}(\mathbf{2 a})$ as dark-red crystalline materials $(0.185 \mathrm{mmol}, 64 \%)$, together with $53 \mathrm{mg}$ of unreacted starting material. The vinylacetylide compound $\mathrm{Cp} * \mathrm{~W}(\mathrm{O}) \mathrm{Re}_{2}\left[\mathrm{CC}(\mathrm{Me})=\mathrm{CH}_{2}\right](\mathrm{CO})_{8}(\mathbf{2 b})$ was prepared under similar conditions in $48 \%$ yield.

Spectral data for $2 a$ : MS (FAB, $\left.{ }^{184} \mathrm{~W},{ }^{187} \mathrm{Re}\right) \mathrm{m} / \mathrm{z} 1034\left(\mathrm{M}^{+}\right)$. IR $\left.\mathrm{C}_{6} \mathrm{H}_{12}\right) v(\mathrm{CO}): 2093(\mathrm{~m}), 2060$ (m), 2004 (vs), 1986 (s), 1979 (s), $1964(w), 1958(w), 1940(v w) \mathrm{cm}^{-1}$. ${ }^{1} \mathrm{H}$ NMR (400 MHz, $\left.\mathrm{CDCl}_{3}, 294 \mathrm{~K}\right): \delta 7.43(\mathrm{t}, 2 \mathrm{H}, \mathrm{J} \mathrm{HH}=7.6 \mathrm{~Hz}), 7.37(\mathrm{~d}, 1 \mathrm{H}, \mathrm{J} \mathrm{HH}$ $=8.0 \mathrm{~Hz}), 7.30(\mathrm{t}, 2 \mathrm{H}, \mathrm{J} \mathrm{нн}=7.2 \mathrm{~Hz}), 2.00\left(\mathrm{~s}, 15 \mathrm{H}, \mathrm{C}_{5} \mathrm{Me}_{5}\right) .{ }^{13} \mathrm{C}$

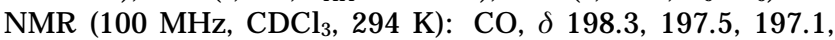
$195.5,191.9,191.5,189.8,187.8 ; \delta 211.4(\mathrm{wc}=77 \mathrm{~Hz}, \mathrm{CCPh})$, 184.6 (CCPh), $143.9\left(1 \mathrm{C}, \mathrm{C}_{6} \mathrm{H}_{5}\right), 129.6\left(2 \mathrm{C}, \mathrm{C}_{6} \mathrm{H}_{5}\right), 128.7$ (1C, $\left.\mathrm{C}_{6} \mathrm{H}_{5}\right), 128.3\left(2 \mathrm{C}, \mathrm{C}_{6} \mathrm{H}_{5}\right), 114.3\left(\mathrm{C}_{5} \mathrm{Me}_{5}\right), 11.3\left(\mathrm{C}_{5} \mathrm{Me}_{5}\right)$. Anal. Calcd for $\mathrm{C}_{26} \mathrm{H}_{20} \mathrm{O}_{9} \mathrm{Re}_{2} \mathrm{~W}_{1}$ : C, 30.24; $\mathrm{H}, 1.95$. Found: C, 30.00, $\mathrm{H} ; 1.96$.

Spectral data for $\mathbf{2 b}$ : MS (FAB, $\left.{ }^{184 \mathrm{~W}},{ }^{187} \mathrm{Re}\right) \mathrm{m} / \mathrm{z} 998\left(\mathrm{M}^{+}\right)$. IR $\left.\mathrm{C}_{6} \mathrm{H}_{12}\right) v(\mathrm{CO}): 2091(\mathrm{~m}), 2060(\mathrm{~m}), 2003$ (vs), $1986(\mathrm{~s}), 1977$ (s), $1964(\mathrm{w}), 1958(\mathrm{w}), 1937(\mathrm{vw}) \mathrm{cm}^{-1}$. ${ }^{1} \mathrm{H}$ NMR $(400 \mathrm{MHz}$, $\left.\mathrm{CDCl}_{3}, 294 \mathrm{~K}\right): \delta 5.22\left(\mathrm{~s}, 1 \mathrm{H}\right.$, cis- $\left.\mathrm{CH}_{2}\right), 5.12\left(\mathrm{~s}, 1 \mathrm{H}\right.$, cis- $\left.\mathrm{CH}_{2}\right)$, $2.12\left(\mathrm{~s}, 15 \mathrm{H}, \mathrm{C}_{5} \mathrm{Me}_{5}\right), 2.00(\mathrm{~s}, 3 \mathrm{H}, \mathrm{Me}) .{ }^{13} \mathrm{C} \mathrm{NMR}(100 \mathrm{MHz}$, $\mathrm{CDCl}_{3}, 294 \mathrm{~K}$ ): $\mathrm{CO}, \delta$ 198.4, 197.7, 197.3, 195.2, 192.3, 191.6, 190.6, 187.8; $\delta 212.8\left(\mathrm{~J} w c=72 \mathrm{~Hz}, \mathrm{C}_{\beta}\right), 181.9\left(\mathrm{C}_{\alpha}\right), 149.1$ $\left(\mathrm{CMe}=\mathrm{CH}_{2}\right), 116.7\left(\mathrm{CMe}=\mathrm{CH}_{2}\right), 114.1\left(\mathrm{C}_{5} \mathrm{Me}_{5}\right), 23.3(\mathrm{Me}), 11.1$ $\left(\mathrm{C}_{5} \mathrm{Me}_{5}\right)$. Anal. Calcd for $\mathrm{C}_{23} \mathrm{H}_{20} \mathrm{O}_{9} \mathrm{Re}_{2} \mathrm{~W}_{1}$ : C, 27.72; $\mathrm{H}, 2.02$. Found: C, 27.52; $\mathrm{H}, 2.01$.

Treatment of $2 \mathbf{a}$ with CO. To a $300 \mathrm{~mL}$ thick-walled glass bottle, the toluene solution $(60 \mathrm{~mL})$ of $\mathbf{2 a}(230 \mathrm{mg}, 0.223 \mathrm{mmol})$ was heated at $90{ }^{\circ} \mathrm{C}$ under a CO atmosphere ( 3 atm) for $2 \mathrm{~h}$ during which the color changed from red to orange. The solvent was evaporated, and the residue was dissolved in $\mathrm{CH}_{2-}$ $\mathrm{Cl}_{2}$ and separated by thin-layer chromatography (dichloromethane:hexane $=1: 2$ ), giving $225 \mathrm{mg}$ of $\mathrm{Cp} * \mathrm{~W}(\mathrm{O}) \mathrm{Re}_{2}-$ $(\mathrm{CCPh})(\mathrm{CO})_{9}(\mathbf{4 a}, 0.212 \mathrm{mmol}, 95 \%)$. Crystals of $\mathbf{4 a}$ suitable for X-ray diffraction study were obtained from dichloromethane and heptane at room temperature. The vinylacetylide complex $\mathrm{Cp} * \mathrm{~W}(\mathrm{O}) \mathrm{Re}_{2}\left[\mathrm{CC}(\mathrm{Me})=\mathrm{CH}_{2}\right](\mathrm{CO})_{9}$ (4) $)$ was prepared under similar conditions.

Spectral data for 4a: MS (FAB, $\left.{ }^{184} \mathrm{~W},{ }^{187} \mathrm{Re}\right) \mathrm{m} / \mathrm{z} 1062\left(\mathrm{M}^{+}\right)$. IR $\left.\mathrm{C}_{6} \mathrm{H}_{12}\right) v(\mathrm{CO}): 2110$ (s), 2052 (m), 2032 (vw), 2014 (vs), 2004 (vs), 1990 (w), 1976 (s), 1966 (w), 1811 (vw, br) cm'-1. IR (KBr) $v(\mathrm{~W} \equiv \mathrm{O}): 930(\mathrm{br}) \mathrm{cm}^{-1}$. ${ }^{1} \mathrm{H}$ NMR $\left(300 \mathrm{MHz} \mathrm{CDCl}_{3}, 294 \mathrm{~K}\right)$ : $\delta 7.72\left(\mathrm{~d}, \mathrm{~J}_{\mathrm{HH}}=7.5 \mathrm{~Hz}, 2 \mathrm{H}\right), 7.50\left(\mathrm{t}, \mathrm{J}_{\mathrm{Hн}}=7.5 \mathrm{~Hz}, 2 \mathrm{H}\right), 7.41$ $(\mathrm{t}, \mathrm{J} \mathrm{HH}=7.5 \mathrm{~Hz}, 1 \mathrm{H}), 1.92(\mathrm{~s}, 15 \mathrm{H}, \mathrm{Cp} *) .{ }^{13} \mathrm{C} \mathrm{NMR}(100.6 \mathrm{MHz}$, $\left.\mathrm{CDCl}_{3}, 294 \mathrm{~K}\right): \mathrm{CO}, \delta 226.0(\mathrm{~J}$ wc $=46 \mathrm{~Hz}, 1 \mathrm{C}), 194.5$ (1C), 192.1 (1C), 190.9 (4C, br), 185.9 (1C), 182.1 (1C, br); $\delta 176.3$ $\left(\mathrm{C}_{\alpha}\right), 147.6\left(\mathrm{~J} \mathrm{wc}=60 \mathrm{~Hz}, \mathrm{C}_{\beta}\right), 135.5\left(\mathrm{i}-\mathrm{C}_{6} \mathrm{H}_{5}\right), 131.3\left(2 \mathrm{C}, \mathrm{o}-\mathrm{C}_{6} \mathrm{H}_{5}\right)$, $\left.128.8\left(2 \mathrm{C}, \mathrm{m}-\mathrm{C}_{6} \mathrm{H}_{5}\right), 128.3\left(\mathrm{p}-\mathrm{C}_{6} \mathrm{H}_{5}\right), 114.0\left(\mathrm{C}_{5} \mathrm{Me}_{5}\right), 10.6\left(\mathrm{C}_{5} \mathrm{Me}\right)_{5}\right)$. Anal. Calcd for $\mathrm{C}_{27} \mathrm{H}_{20} \mathrm{O}_{10} \mathrm{Re}_{2} \mathrm{~W}$ : C, 30.57; $\mathrm{H}, 1.90$. Found: C, 30.41, H, 1.96 .

Spectral data for $\mathbf{4 b}$ : MS (FAB, $\left.{ }^{184} \mathrm{~W},{ }^{187} \mathrm{Re}\right) \mathrm{m} / \mathrm{z} 1026\left(\mathrm{M}^{+}\right)$. IR $\left.\mathrm{C}_{6} \mathrm{H}_{12}\right) v(\mathrm{CO}): 2110(\mathrm{~s}), 2052$ (m), 2031 (vw), 2014 (vs), 2005 (vs), 1988 (w), 1976 (s), 1965 (w), 1808 (vw, br) cm ${ }^{-1}$. ${ }^{1} \mathrm{H}$ NMR $\left(300 \mathrm{MHz} \mathrm{CDCl}_{3}, 294 \mathrm{~K}\right): \delta 5.54(\mathrm{~s}, 1 \mathrm{H}), 5.46(\mathrm{~s}, 1 \mathrm{H}), 2.37$ (s, $3 \mathrm{H}, \mathrm{Me}), 2.01$ (s, 15H, Cp*). ${ }^{13} \mathrm{C}$ NMR (100.6 $\mathrm{MHz} \mathrm{CDCl}_{3}$, $294 \mathrm{~K}$ ): CO, $\delta 226.6(\mathrm{~J} \mathrm{wc}=42 \mathrm{~Hz}, 1 \mathrm{C}), 194.5(1 \mathrm{C}), 191.9$ (1C), 191.0 (4C, br), 186.1 (1C), $182.2(1 \mathrm{C}, \mathrm{br}) ; \delta 175.6\left(\mathrm{C}_{\alpha}\right), 148.8$ $\left(\mathrm{J} w \mathrm{wc}=61 \mathrm{~Hz}, \mathrm{C}_{\beta}\right), 138.8\left(\mathrm{C}_{\gamma}\right), 119.7\left(\mathrm{CH}_{2}\right), 114.1\left(\mathrm{C}_{5} \mathrm{Me}_{5}\right), 25.3$ (Me), 10.7 ( $\left.\mathrm{C}_{5} \mathrm{Me}_{5}\right)$. Anal. Calcd for $\mathrm{C}_{24} \mathrm{H}_{20} \mathrm{O}_{10} \mathrm{Re}_{2} \mathrm{~W}: \mathrm{C}, 28.13$, $\mathrm{H}$; 1.97. Found: C, 28.08; $\mathrm{H}, 2.10$.

Thermolysis of $\mathbf{4 a}$. A toluene solution $(30 \mathrm{~mL})$ of $\mathbf{4 a}(26.6$ $\mathrm{mg}, 0.026 \mathrm{mmol}$ ) was refluxed under nitrogen for $3 \mathrm{~h}$, during which the color changed from orange to red. After removal of the solvent, the residue was dissolved in $\mathrm{CH}_{2} \mathrm{Cl}_{2}$ and then separated using thin-layer chromatography (dichloromethane: hexane $=1: 2)$, giving $21.5 \mathrm{mg}$ of $\mathbf{2 a}(0.022 \mathrm{mmol}, 83 \%)$.

Hydrogenation of $\mathbf{2 a}$. A toluene solution $(60 \mathrm{~mL})$ of $\mathbf{2 a}$ (150 mg, $0.145 \mathrm{mmol}$ ) was heated at reflux under hydrogen (1 atm) for $1.5 \mathrm{~h}$, during which the color changed from orange to red-brown. After the solution was allowed to reach room 
temperature, the solvent was evaporated and the residue was taken in $\mathrm{CH}_{2} \mathrm{Cl}_{2}$ and separated by TLC (dichloromethane: hexane $=1: 1)$, giving $31 \mathrm{mg}$ of dark-red $\mathrm{Cp}^{* W \mathrm{Re}_{2}}(\mu-\mathrm{O})(\mu-\mathrm{H})_{2^{-}}$ $(\mathrm{CCPh})(\mathrm{CO})_{6}(5 \mathrm{a}, 0.032 \mathrm{mmol}, 22 \%), 39 \mathrm{mg}$ of orange $\mathrm{Cp} * \mathrm{~W}$ (O) $\operatorname{Re}_{2}(\mu-\mathrm{CHCHPh})(\mathrm{CO})_{8}(6 \mathbf{6}, 0.038 \mathrm{mmol}, 26 \%)$, and $22 \mathrm{mg}$ of yellow Cp*W(O)Re $2(u-\mathrm{H})\left(u-\mathrm{CHCH}_{2} \mathrm{Ph}\right)(\mathrm{CO})_{8}(7 \mathrm{a}, 0.021 \mathrm{mmol}$, $14 \%)$. Crystals of $\mathbf{6 a}$ and $7 \mathbf{7 a}$ suitable for X-ray diffraction study were recrystallized at room temperature from a layered solution of chloroform-hexane and chloroform-methanol, respectively.

In an another experiment, a heptane solution (30 mL) of $2 \mathbf{a}$ ( $25 \mathrm{mg}, 0.024 \mathrm{mmol}$ ) was heated at reflux under hydrogen (1 atm) for $40 \mathrm{~min}$. After removal of the heptane in vacuo, the residue was dissolved in a minimum amount of $\mathrm{CH}_{2} \mathrm{Cl}_{2}$ and passed through a short silica gel column to remove the trace amount of decomposition product. The ${ }^{1} \mathrm{H}$ NMR analysis indicated that the relative yields of complexes $\mathbf{2 a}, \mathbf{5 a}, \mathbf{6 a}$, and 7a were $12 \%, 40 \%, 24 \%$, and $5 \%$, respectively.

Spectral data for 5a: MS (FAB, $\left.{ }^{184} \mathrm{~W},{ }^{187} \mathrm{Re}\right) \mathrm{m} / \mathrm{z} 980\left(\mathrm{M}^{+}\right)$. IR $\left(\mathrm{C}_{6} \mathrm{H}_{12}\right) v(\mathrm{CO}): 2052$ (vs), 2017 (vs), 1971 (vs), 1954 (vs), 1938 (m), 1932 (s) cm ${ }^{-1}$. ${ }^{1} \mathrm{H}$ NMR (400 MHz, CDCl $3,294 \mathrm{~K}$ ): $\delta 7.92(\mathrm{~d}, 2 \mathrm{H}, \mathrm{J} \mathrm{HH}=8 \mathrm{~Hz}), 7.53\left(\mathrm{t}, 2 \mathrm{H}, \mathrm{J}_{\mathrm{HH}}=7.2 \mathrm{~Hz}\right), 7.38(\mathrm{t}$, $1 \mathrm{H}, \mathrm{J} \mathrm{HH}=7.2 \mathrm{~Hz}), 2.09\left(\mathrm{~s}, 15 \mathrm{H}, \mathrm{C}_{5} \mathrm{Me}_{5}\right),-7.44(\mathrm{~s}, 1 \mathrm{H}, \mathrm{J} \mathrm{wH}=$ $135 \mathrm{~Hz}),-14.08(\mathrm{~s}, 1 \mathrm{H}) .{ }^{13} \mathrm{C}$ NMR $\left(100 \mathrm{MHz} \mathrm{CDCl}_{3}, 294 \mathrm{~K}\right)$ : CO, $\delta 194.5$ (br), 193.9, 189.9, $189.0(\mathrm{br}), 188.1,184.2(\mathrm{br}) ; \delta$ 156.6 (CCPh), 137.4, 132.4 (2C), 129.8, 128.9 (2C), 121.6 (J wc $=71 \mathrm{~Hz}, \mathrm{CCPh}), 114.3\left(\mathrm{C}_{5} \mathrm{Me}_{5}\right), 11.6\left(\mathrm{C}_{5} \mathrm{Me}_{5}\right)$. Anal. Calcd for $\mathrm{C}_{24} \mathrm{H}_{22} \mathrm{O}_{7} \mathrm{Re}_{2} \mathrm{~W}_{1} \cdot 1 / 2 \mathrm{CHCl}_{3}$ : C, 28.34; $\mathrm{H}, 2.18$. Found: $\mathrm{C}$, 28.28; $\mathrm{H}, 2.25$.

Spectral data for 6a: $M S\left(F A B,{ }^{184} \mathrm{~W},{ }^{187} \mathrm{Re}\right) \mathrm{m} / \mathrm{z} 1036\left(\mathrm{M}^{+}\right)$. IR $\mathrm{C}_{6} \mathrm{H}_{12}$ ) $v(\mathrm{CO}): 2088$ (m), 2043 (s), 2008 (vs), 1992 (vs), 1981 (s), 1961 (vw), 1942 (w), 1919 (s) cm $\mathrm{cm}^{-1} \mathrm{H}$ NMR (400 MHz, $\left.\mathrm{CDCl}_{3}, 294 \mathrm{~K}\right): \delta 7.42-7.21\left(\mathrm{~m}, 5 \mathrm{H}, \mathrm{C}_{6} \mathrm{H}_{5}\right), 5.93(\mathrm{~d}, 1 \mathrm{H}, 3 \mathrm{\jmath н}$ $=13.6 \mathrm{~Hz}), 4.72\left(\mathrm{~d}, 1 \mathrm{H}, 3 \mathrm{~J}_{\mathrm{HH}}=13.6 \mathrm{~Hz}\right), 2.22\left(\mathrm{~s}, 15 \mathrm{H}, \mathrm{C}_{5} \mathrm{Me}_{5}\right)$ ${ }^{13} \mathrm{C}$ NMR $\left(100 \mathrm{MHz} \mathrm{CDCl}_{3}, 294 \mathrm{~K}\right): \mathrm{CO}, \delta 207.3,205.4,202.3$, 196.3, 189.7, 189.1, 186.2, 183.5; $\delta 161.3(\mathrm{~J} \mathrm{wc}=115 \mathrm{~Hz}$, CHCHPh), 142.9, $129.4(2 \mathrm{C}), 127.9,125.7(2 \mathrm{C}), 112.2\left(\mathrm{C}_{5} \mathrm{Me}_{5}\right)$, 76.8 ( $\mathrm{CHCHPh}$ ), $12.3\left(\mathrm{C}_{5} \mathrm{Me}\right)$ ). Anal. Calcd for $\mathrm{C}_{27} \mathrm{H}_{24} \mathrm{O}_{9} \mathrm{Re}_{2}-$ $\mathrm{W}_{1} \mathrm{Cl}_{2}$ : C, 28.96; $\mathrm{H}, 2.16$. Found: C, 29.39; $\mathrm{H}, 2.18$.

Spectral data for 7a: MS (FAB, $\left.{ }^{184}{ }^{\mathrm{W}},{ }^{187} \mathrm{Re}\right) \mathrm{m} / \mathrm{z} 1038\left(\mathrm{M}^{+}\right)$. IR $\left.\mathrm{C}_{6} \mathrm{H}_{12}\right) v(\mathrm{CO}) ; 2087$ (s), 2041 (s), 2000 (vs), 1987 (vs), 1974 (w), $1963(w), 1943(w, b r) \mathrm{cm}^{-1}$. ${ }^{1} \mathrm{H}$ NMR (300 MHz, CDCl 3 , $294 \mathrm{~K}): \delta 7.42-7.30\left(\mathrm{~m}, 5 \mathrm{H}, \mathrm{C}_{6} \mathrm{H}_{5}\right), 7.15(\mathrm{dd}, 1 \mathrm{H}, 3 \mathrm{\jmath} \mathrm{Hн}=9.8$ and $5.5 \mathrm{~Hz}), 4.65\left(\mathrm{dd}, 1 \mathrm{H},{ }^{2} \mathrm{~J} \mathrm{HH}=14 \mathrm{~Hz},{ }^{3} \mathrm{~J} \mathrm{HH}=5.5 \mathrm{~Hz}\right), 4.13$ $\left(\mathrm{d}, 1 \mathrm{H},{ }^{2} \mathrm{~J} \mathrm{нн}=14 \mathrm{~Hz},{ }^{3} \mathrm{Jн}=9.8 \mathrm{~Hz}\right), 2.08\left(\mathrm{~s}, 15 \mathrm{H}, \mathrm{C}_{5} \mathrm{Me}_{5}\right)$, $-10.93\left(\mathrm{~s}, 1 \mathrm{H}, \mathrm{J}_{\mathrm{w}-\mathrm{H}}=97.2 \mathrm{~Hz}\right) .{ }^{13} \mathrm{C} \mathrm{NMR}\left(75 \mathrm{MHz} \mathrm{CDCl}_{3}\right.$, $294 \mathrm{~K})$ : CO, $\delta$ 198.3, 193.9, 193.8, 193.1, 192.3, 191.2, 190.8, 190.1; $\delta$ 146.7, $141.6\left(\mathrm{~J} w \mathrm{w}=104 \mathrm{~Hz}, \mathrm{CHCH}_{2} \mathrm{Ph}\right), 128.6$ (2C), $128.5(2 \mathrm{C}), 126.7,114.0\left(\mathrm{C}_{5} \mathrm{Me}_{5}\right), 61.7\left(\mathrm{CHCH}_{2} \mathrm{Ph}\right), 11.6\left(\mathrm{C}_{5} \mathrm{Me}\right)$. Anal. Calcd for $\mathrm{C}_{26} \mathrm{H}_{24} \mathrm{O}_{9} \mathrm{Re}_{2} \mathrm{~W}_{1}$ : C, 30.12; $\mathrm{H}, 2.33$. Found: C, 29.96; $\mathrm{H}, 2.35$.

Treatment of 5 a with CO. A heptane solution $(30 \mathrm{~mL})$ of $5 \mathbf{a}(13 \mathrm{mg}, 0.013 \mathrm{mmol}$ ) was heated at reflux under CO (1 atm) for $2 \mathrm{~min}$, during which the color changed from yellow to redorange. The solvent was then evaporated, the residue was taken up in $\mathrm{CH}_{2} \mathrm{Cl}_{2}$ and separated by thin-layer chromatography (dichloromethane:hexane $=1: 2$ ), giving $2.3 \mathrm{mg}$ of $\mathbf{2 a}$ $(0.0022 \mathrm{mmol}, 17 \%)$ and $2.7 \mathrm{mg}$ of $\mathbf{6 a}(0.0026 \mathrm{mmol}, 20 \%)$.

Hydrogenation of $\mathbf{6 a}$. A toluene solution $(25 \mathrm{~mL})$ of $\mathbf{6 a}$ (22 $\mathrm{mg}, 0.021 \mathrm{mmol}$ ) was heated under hydrogen ( $3 \mathrm{~atm}$ ) in a pressure bottle at $85{ }^{\circ} \mathrm{C}$ for $1.5 \mathrm{~h}$, during which the color changed from yellow-orange to red-orange. After the solution was cooled to room temperature, the solvent was evaporated and the residue was dissolved in a minimum amount of $\mathrm{CH}_{2-}$ $\mathrm{Cl}_{2}$ and separated by thin-layer chromatography (dichloromethane:hexane $=1: 2)$, giving $2.3 \mathrm{mg}$ of $\mathbf{6 a}(0.0022 \mathrm{mmol}$, $10 \%)$ and $9.2 \mathrm{mg}$ of yellow $7 \mathrm{a}(0.0088 \mathrm{mmol}, 42 \%)$ as the major products. The deuterium labeled compound $7 \mathbf{a}$ with formula $\mathrm{Cp}^{*} \mathrm{~W}(\mathrm{O}) \mathrm{Re}_{2}(\mu-\mathrm{D})(\mu-\mathrm{CHCHDPh})(\mathrm{CO})_{8}$ was prepared from the reaction with $\mathrm{D}_{2}$ under similar conditions.

Thermolysis of $\mathbf{6 a}$. A toluene solution $(15 \mathrm{~mL})$ of $\mathbf{6 a}(9.5$ $\mathrm{mg}, 0.009 \mathrm{mmol}$ ) was refluxed under a nitrogen atmosphere (1 atm) for $2 \mathrm{~h}$. After removal of the solvent, the residue was taken up in $\mathrm{CH}_{2} \mathrm{Cl}_{2}$ and separated by thin-layer chromatography (dichloromethane:hexane $=1: 2$ ), giving $0.3 \mathrm{mg}$ of $\mathbf{2 a}$ (0.004 $\mathrm{mmol}, 3 \%)$ and $3.1 \mathrm{mg}$ of $5 \mathrm{a}(0.004 \mathrm{mmol}, 35 \%)$, in addition to $4.2 \mathrm{mg}$ of unreacted $\mathbf{6 a}(0.009 \mathrm{mmol}, 44 \%)$.

Thermolysis of 7a. A toluene solution (30 mL) of $7 \mathbf{7 a}(36$ $\mathrm{mg}, 0.034 \mathrm{mmol}$ ) was refluxed under nitrogen for $2 \mathrm{~h}$, during which the color changed from yellow to orange. After removal of the solvent, the residue was taken up in $\mathrm{CH}_{2} \mathrm{Cl}_{2}$ and separated by thin-layer chromatography (dichloromethane: hexane = 1:2), giving $4.1 \mathrm{mg}$ of $\mathbf{5 a}(0.004 \mathrm{mmol}, 12 \%), 19 \mathrm{mg}$ of $\mathbf{6 a}(0.019 \mathrm{mmol}, 55 \%)$, and $9.2 \mathrm{mg}$ of unreacted $7 \mathbf{a}(0.009$ $\mathrm{mmol}$, 25\%).

Hydrogenation of $\mathbf{2 b}$. A toluene solution $(50 \mathrm{~mL})$ of $\mathbf{2 b}$ (50.4 $\mathrm{mg}, 0.051 \mathrm{mmol}$ ) was heated at reflux under hydrogen (1 atm) for $30 \mathrm{~min}$, during which the color changed from orange to red-brown. After removal of the solvent, the residue was taken up in $\mathrm{CH}_{2} \mathrm{Cl}_{2}$ and separated by thin-layer chromatography (dichloromethane:hexane $=1: 2$ ), giving $2 \mathrm{mg}$ of yellow $\mathrm{Cp} * \mathrm{~W}(\mathrm{O}) \mathrm{Re}_{2}(u-\mathrm{H})\left[\mu-\mathrm{CHCH}_{2} \mathrm{C}(\mathrm{Me})=\mathrm{CH}_{2}\right](\mathrm{CO})_{8}(\mathbf{7 b}, 0.002 \mathrm{mmol}$, $4 \%), 16 \mathrm{mg}$ of brown Cp*WRe$(u-\mathrm{O})(\mu-\mathrm{H})_{2}\left[\mu-\mathrm{CCC}(\mathrm{Me})=\mathrm{CH}_{2}\right]-$ $(\mathrm{CO})_{6}(5 \mathbf{b}, 0.016 \mathrm{mmol}, 32 \%), 7.5 \mathrm{mg}$ of orange $\mathrm{Cp} * \mathrm{~W}(\mathrm{O}) \mathrm{Re}_{2-}$ $\left[\mu-\mathrm{CHCHC}(\mathrm{Me})=\mathrm{CH}_{2}\right](\mathrm{CO})_{8}(\mathbf{6 b}, 0.008 \mathrm{mmol}, 15 \%)$, and $8.7 \mathrm{mg}$ of red-brown $\mathrm{Cp}^{*} \mathrm{WRe}_{2}(u-\mathrm{O})\left[\mu-\mathrm{CHCC}(\mathrm{Me})_{2}\right](\mathrm{CO})_{7} \quad(\mathbf{8}, 0.009$ $\mathrm{mmol}, 18 \%)$, in the order of their elution. Crystals of $\mathbf{8}$ suitable for X-ray diffraction study were obtained from a layered solution of chloroform-heptane at room temperature.

Spectral data for 5b: MS (FAB, $\left.{ }^{184} \mathrm{~W},{ }^{187} \mathrm{Re}\right) \mathrm{m} / \mathrm{z} 944\left(\mathrm{M}^{+}\right)$. IR $\left.\mathrm{C}_{6} \mathrm{H}_{12}\right) v(\mathrm{CO}): 2052$ (s), 2017 (vs), 1970 (s, br), 1954 (s, br), 1938 (m, br), $1932(\mathrm{~m}, \mathrm{br}) \mathrm{cm}^{-1}$. ${ }^{1} \mathrm{H}$ NMR (300 MHz, CDCl 3 , $294 \mathrm{~K}): \delta 5.72\left(1 \mathrm{H}, \mathrm{CH}_{2}\right), 5.65\left(1 \mathrm{H}, \mathrm{CH}_{2}\right), 2.41(\mathrm{~s}, 3 \mathrm{H}, \mathrm{Me})$, $2.11(\mathrm{~s}, 15 \mathrm{H}, \mathrm{Cp} *),-7.59(\mathrm{~s}, \mathrm{~J} \mathrm{wH}=135.6 \mathrm{~Hz}, 1 \mathrm{H}),-14.18(\mathrm{~s}$, $1 \mathrm{H})$.

Spectral data for $6 \mathbf{b}:$ MS (FAB, $\left.{ }^{184} \mathrm{~W},{ }^{187} \mathrm{Re}\right) \mathrm{m} / \mathrm{z} 1000\left(\mathrm{M}^{+}\right)$. IR $\left.\mathrm{C}_{6} \mathrm{H}_{12}\right) v(\mathrm{CO}): 2088(\mathrm{~m}), 2043$ (s), 2007 (vs), 1989 (vs), 1980 (s), 1961 (vw, br), 1942 (w, br), 1918 (s) cm ${ }^{-1} .{ }^{1} \mathrm{H}$ NMR (300 $\left.\mathrm{MHz}, \mathrm{CDCl}_{3}, 294 \mathrm{~K}\right): \delta 5.41(\mathrm{~s}, 1 \mathrm{H}), 5.35(\mathrm{~s}, 1 \mathrm{H}), 5.22(\mathrm{~d}, \mathrm{~J} \mathrm{HH}$ $\left.=13.7 \mathrm{~Hz}, 1 \mathrm{H}, \mathrm{C}_{\alpha} \mathrm{H}\right), 4.60\left(\mathrm{~d}, \mathrm{~J}\right.$ нн $\left.=13.7 \mathrm{~Hz}, 1 \mathrm{H}, \mathrm{C}_{\beta} \mathrm{H}\right), 2.18$ (s, $\left.15 \mathrm{H}, \mathrm{Cp}^{*}\right), 1.61(\mathrm{~s}, 3 \mathrm{H}, \mathrm{Me}) .{ }^{13} \mathrm{C}$ NMR $\left(75.5 \mathrm{MHz} \mathrm{CDCl}_{3}\right.$, $294 \mathrm{~K})$ : CO, $\delta$ 207.4, 205.5, 202.3, 196.4, 189.9, 188.9, 186.5, 183.8; $\delta 160.2\left(\mathrm{~J} \mathrm{wc}=114 \mathrm{~Hz}, \mathrm{C}_{\alpha} \mathrm{H}\right), 145.3\left(\mathrm{C}_{\gamma}\right), 118.1\left(\mathrm{C}_{5} \mathrm{Me}_{5}\right)$, $112.0\left(\mathrm{C}_{\beta} \mathrm{H}\right), 84.4\left(\mathrm{CH}_{2}\right), 16.4(\mathrm{Me}), 12.2\left(\mathrm{C}_{5} \mathrm{Me}_{5}\right)$. Anal. Calcd for $\mathrm{C}_{23} \mathrm{H}_{22} \mathrm{O}_{9} \mathrm{Re}_{2} \mathrm{~W}: \mathrm{C}, 27.66 ; \mathrm{H}, 2.22$. Found: $\mathrm{C}, 27.52 ; \mathrm{H}, 2.28$.

Spectral data for $7 \mathbf{b}$ : $M S\left(F A B,{ }^{184} \mathrm{~W},{ }^{187} \mathrm{Re}\right) \mathrm{m} / \mathrm{z} 1002\left(\mathrm{M}^{+}\right)$. IR $\left.\mathrm{C}_{6} \mathrm{H}_{12}\right) v(\mathrm{CO}): 2087$ (s), 2041 (vs), 2001 (vs), 1987 (vs, br), 1962 (w), 1942 (s, br) cm ${ }^{-1}$. ${ }^{1} \mathrm{H}$ NMR (400 MHz, CDCl, 294 $\mathrm{K}): \delta 7.13\left(\mathrm{dd}, \mathrm{J}_{\mathrm{HH}}=11.2\right.$ and $\left.3.2 \mathrm{~Hz}, 1 \mathrm{H}\right), 4.97(\mathrm{~s}, 1 \mathrm{H}), 4.91$ $(\mathrm{s}, 1 \mathrm{H}), 4.05\left(\mathrm{~d}, \mathrm{~J}_{\mathrm{Hн}}=14.8\right.$ and $\left.3.2 \mathrm{~Hz}, 1 \mathrm{H}\right), 3.57\left(\mathrm{dd}, \mathrm{J}_{\mathrm{Hн}}=\right.$ 14.8 and $11.2 \mathrm{~Hz}, 1 \mathrm{H}$ ), 2.07 (s, 15H, Cp*), 2.00 (s, 3H, Me), $-10.84(\mathrm{~s}, \mathrm{~J} \mathrm{wH}=97.6 \mathrm{~Hz}, 1 \mathrm{H}) .{ }^{13} \mathrm{C} \mathrm{NMR}\left(75.5 \mathrm{MHz} \mathrm{CDCl}_{3}\right.$, 294 K): CO, $\delta 199.3$ (br), 194.0, 193.8 (br), 193.0, 192.3, 191.2 (br), 190.7, 190.1; $\delta 149.1\left(\mathrm{C}_{\gamma}\right), 137.0\left(\mathrm{~J} \mathrm{wc}=104 \mathrm{~Hz}, \mathrm{C}_{\alpha} \mathrm{H}\right)$, $113.8\left(\mathrm{C}_{5} \mathrm{Me}_{5}\right), 112.4\left(\mathrm{CH}_{2}\right), 63.7\left(\mathrm{C}_{\beta}\right), 21.8(\mathrm{Me}), 11.6\left(\mathrm{C}_{5} \mathrm{Me}_{5}\right)$. Anal. Calcd for $\mathrm{C}_{23} \mathrm{H}_{24} \mathrm{O}_{9} \mathrm{Re}_{2} W$ : C, 27.61; H, 2.42. Found: $\mathrm{C}$, 27.43; $\mathrm{H}, 2.41$.

Spectral data for 8: MS (FAB, $\left.{ }^{184} \mathrm{~W},{ }^{187} \mathrm{Re}\right) \mathrm{m} / \mathrm{z} 972\left(\mathrm{M}^{+}\right)$. IR $\left.\mathrm{C}_{6} \mathrm{H}_{12}\right) v(\mathrm{CO}): 2046$ (s), 2010 (vs), 1972 (s), 1963 (w), 1936 (s), 1919 (w), $1777(\mathrm{vw}, \mathrm{br}) \mathrm{cm}^{-1}$. ${ }^{1} \mathrm{H} \mathrm{NMR}\left(300 \mathrm{MHz} \mathrm{CDCl}_{3}\right.$, $294 \mathrm{~K}): \delta 9.23\left(\mathrm{~s}, 1 \mathrm{H}, \mathrm{C}_{\alpha} \mathrm{H}\right), 2.22(\mathrm{~s}, 3 \mathrm{H}, \mathrm{Me}), 2.09(\mathrm{~s}, 15 \mathrm{H}$, Cp*), 1.79 (s, 3H, Me). ${ }^{13} \mathrm{C}$ NMR (100.6 MHz, CDCl $\left.3,294 \mathrm{~K}\right)$ : CO, $\delta 251.9(\mathrm{~J} w \mathrm{wc}=133 \mathrm{~Hz}, 1 \mathrm{C}), 200.0$ (1C), 197.4 (4C), 188.9 (1C); $\delta 165.0\left(\mathrm{C}_{\beta}\right), 136.0\left(\mathrm{wc}=97 \mathrm{~Hz}, \mathrm{C}_{\alpha} \mathrm{H}\right), 112.7\left(\mathrm{C}_{5} \mathrm{Me}_{5}\right)$, $66.0\left(\mathrm{C}_{\gamma}\right), 27.4(2 \mathrm{Me}), 10.1$ ( $\mathrm{C}_{5} \mathrm{Me}$ ). Anal. Calcd for $\mathrm{C}_{22} \mathrm{H}_{22}$ $\mathrm{O}_{8} \mathrm{Re}_{2} \mathrm{~W}$ : C, 27.22; H, 2.28. Found: C, 27.11; H, 2.26.

Hydrogenation of $\mathbf{6 b}$. To a $300 \mathrm{~mL}$ glass pressure bottle, toluene $(30 \mathrm{~mL})$ and $\mathbf{6 b}(25.8 \mathrm{mg}, 0.026 \mathrm{mmol})$ were added. The bottle was charged with $3 \mathrm{~atm}$ of hydrogen and placed into a preheated oil bath maintained at $90{ }^{\circ} \mathrm{C}$ for $2 \mathrm{~h}$. After removal of the solvent, the residue was taken up in $\mathrm{CH}_{2} \mathrm{Cl}_{2}$ and separated by thin-layer chromatography (dichloromethane: hexane $=1: 2)$, producing $4 \mathrm{mg}$ of yellow $\mathbf{7 b}(0.004 \mathrm{mmol}, 15 \%)$ together with $12.8 \mathrm{mg}$ of $\mathbf{6 b}$.

Thermolysis of $\mathbf{7 b}$. A toluene solution $(30 \mathrm{~mL})$ of $\mathbf{7 b}$ (10.8 $\mathrm{mg}, 0.011 \mathrm{mmol}$ ) was heated at reflux under nitrogen for $2 \mathrm{~h}$. 
Table 1. Experimental Data for the X-ray Diffraction Studies of Complexes $4 a, 6 a, 7 a$, and $8^{a}$

\begin{tabular}{|c|c|c|c|c|}
\hline & $4 a$ & $6 a$ & $7 a$ & 8 \\
\hline $\begin{array}{l}\text { formula } \\
\text { mw }\end{array}$ & $\begin{array}{l}\mathrm{C}_{27} \mathrm{H}_{20} \mathrm{O}_{10} \mathrm{Re}_{2} W \\
1060.71\end{array}$ & $\begin{array}{l}\mathrm{C}_{26} \mathrm{H}_{22} \mathrm{O}_{9} \mathrm{Re}_{2} \mathrm{~W} \cdot 1 / 2 \mathrm{CH}_{2} \mathrm{Cl}_{2} \\
1077.18\end{array}$ & $\begin{array}{l}\mathrm{C}_{26} \mathrm{H}_{24} \mathrm{O}_{9} \mathrm{Re}_{2} \mathrm{~W} \\
1036.73\end{array}$ & $\mathrm{C}_{22} \mathrm{H}_{22} \mathrm{O}_{8} \mathrm{Re}_{2} W$ \\
\hline cryst syst & trigonal & monoclinic & orthorhombic & monoclinic \\
\hline space group & $\mathrm{R} \overline{3}$ & $\mathrm{C} 2 / \mathrm{c}$ & $\mathrm{P} 2_{1} 2_{1} 2_{1}$ & $\mathrm{P} 2_{1} / \mathrm{n}$ \\
\hline $\mathrm{a}(\AA)$ & $41.77(1)$ & $16.847(5)$ & $11.743(4)$ & $10.542(2)$ \\
\hline $\mathrm{b}(\AA)$ & & $16.303(5)$ & $12.922(4)$ & $14.713(3)$ \\
\hline$c(\AA)$ & $9.386(5)$ & $23.044(4)$ & 18.794(4) & $16.138(4)$ \\
\hline$\beta$ (deg) & & $111.26(2)$ & & $98.62(2)$ \\
\hline volume $\left(\AA^{3}\right)$ & 14 179(8) & $5898(3)$ & 2852(1) & 2475(1) \\
\hline Z & 18 & 8 & 4 & 4 \\
\hline$D_{c}\left(\mathrm{~g} / \mathrm{cm}^{3}\right)$ & 2.236 & 2.426 & 2.415 & 2.605 \\
\hline$F(000)$ & 8748 & 3960 & 1904 & 1768 \\
\hline $2 \theta(\max )$ & $50^{\circ}$ & $45^{\circ}$ & $50^{\circ}$ & $50^{\circ}$ \\
\hline$h, k, I$ ranges & -41 to $42,0-49,0-11$ & -18 to $16,0-17,0-24$ & -0 to $13,0-15,0-22$ & -12 to $12,0-17,0-19$ \\
\hline cryst size (mm) & $0.20 \times 0.20 \times 0.50$ & $0.25 \times 0.35 \times 0.45$ & $0.20 \times 0.25 \times 0.40$ & $0.13 \times 0.25 \times 0.35$ \\
\hline$\mu(\operatorname{MoK} \alpha)\left(\mathrm{cm}^{-1}\right)$ & 115.40 & 123.18 & 127.39 & 146.76 \\
\hline transmission: $\max , \min$ & $1.00,0.47$ & $1.00,0.67$ & $1.00,0.38$ & $1.00,0.41$ \\
\hline no. of unique data & 5545 & 3859 & 2826 & 4344 \\
\hline no. of data with I $>2 \sigma(I)$ & 3695 & 3250 & 2491 & 3474 \\
\hline no. of atoms, params & 60,362 & 63,358 & 61,344 & 55,299 \\
\hline weight modifier (g) & 0.00001 & unit weight & unit weight & unit weight \\
\hline maximum $\Delta / \sigma$ ratio & 0.056 & 0.010 & 0.045 & 0.006 \\
\hline $\mathrm{R}_{\mathrm{F}} ; \mathrm{R}_{\mathrm{w}}$ & $0.040 ; 0.047$ & $0.025 ; 0.022$ & $0.029 ; 0.023$ & $0.036 ; 0.031$ \\
\hline GOF & 2.90 & 1.87 & 1.66 & 2.47 \\
\hline diff map, max/min $\left(e / \AA^{3}\right)$ & $1.49 /-1.13$ & $2.42 /-0.72$ & $1.30 /-0.80$ & $1.30 /-1.87$ \\
\hline
\end{tabular}

a Features common to all determinations: Nonius CAD-4 diffractometer, $\lambda\left(M_{0} K_{\alpha}\right)=0.7107 \AA$; minimize function: $\sum\left(w \mid F_{0}-F_{c}{ }^{2}\right)$, weighting scheme: $\mathrm{w}^{-1}=\sigma^{2}\left(\mathrm{~F}_{0}\right)+|\mathrm{g}| \mathrm{F}_{0}^{2} ; \mathrm{GOF}=\left[\Sigma \mathrm{w}\left|\mathrm{F}_{\mathrm{o}}-\mathrm{F}_{\mathrm{c}}\right|^{2} /\left(\mathrm{N}_{\mathrm{o}}-\mathrm{N}_{\mathrm{v}}\right)\right]^{1 / 2}\left(\mathrm{n}_{\mathrm{o}}=\right.$ number of observations; $\mathrm{N}_{\mathrm{v}}=$ number of variables).

After removal of the solvent in vacuo, the oily residue was dissolved in dichloromethane and passed through a short silica gel column to remove trace amount of decomposition product. The ${ }^{1} \mathrm{H}$ NMR analysis indicated that the relative yields of $\mathbf{6 b}$, 7b, and $\mathbf{8}$ were $5 \%, 13 \%$, and $15 \%$, respectively.

Thermolysis of $\mathbf{6 b}$. A toluene solution $(30 \mathrm{~mL})$ of $\mathbf{6 b}(23.8$ $\mathrm{mg}, 0.024 \mathrm{mmol}$ ) was heated at reflux under nitrogen for $2 \mathrm{~h}$. After removal of the solvent, the residue was taken up in $\mathrm{CH}_{2-}$ $\mathrm{Cl}_{2}$ and separated by thin-layer chromatography (dichloromethane:hexane $=1: 2)$, giving $5.5 \mathrm{mg}$ of red-brown 8 (0.0057 $\mathrm{mmol}, 24 \%$ ) and $4.3 \mathrm{mg}$ of unreacted $\mathbf{6 b}$.

X-ray Crystallography. The X-ray diffraction measurements were carried out on a Nonius CAD-4 diffractometer at room temperature. Lattice parameters were determined from 25 randomly selected high-angle reflections. Three standard reflections were monitored every $3600 \mathrm{~s}$. No significant change in intensities, due to crystal decay, was observed over the course of all data collection. Intensities of the diffraction signals were corrected for L orentz, polarization, and absorption effects ( $\psi$ scans). The structure was solved by using the NRCC-SDP-VAX package. All the non-hydrogen atoms had anisotropic temperature factors, while the hydrogen atoms of the organic substituents were placed at the calculated positions with $U_{H}=U_{C}+0.1$. The experimental data of the X-ray diffraction studies of $\mathbf{2} \mathbf{b}$ and $\mathbf{5 a}$ have been reported in a previous communication; therefore, these data were not duplicated in this article. ${ }^{6}$ The refinement parameters of complexes 4a, 6a, 7a and 8 are given in Table 1, while their selected bond distances and angles are presented in Tables $2-5$, respectively.

\section{Results}

Syntheses and Characterization of Oxo-Acetylide Complexes 2 and 4. Treatment of $\mathrm{Cp}^{*} \mathrm{WRe} \mathrm{C}_{2}$ $(\mathrm{CCR})(\mathrm{CO})_{9}\left(\mathbf{l a}, \mathrm{R}=\mathrm{Ph} ; \mathbf{1 b}, \mathrm{R}=\mathrm{C}(\mathrm{Me})=\mathrm{CH}_{2}\right)$ with oxygen or nitrous oxide gave the oxo compound $\mathrm{Cp} * \mathrm{~W}(\mathrm{O})$ $\left(\mathrm{Re}_{2} \mathrm{CCR}\right)(\mathrm{CO})_{8}\left(\mathbf{2 a}, \mathrm{R}=\mathrm{Ph} ; \mathbf{2 b}, \mathrm{R}=\mathrm{C}(\mathrm{Me})=\mathrm{CH}_{2}\right)$ in $48-64 \%$ yields, based on the starting materials consumed. These compounds have been characterized by a combination of microanalysis, solution IR studies ${ }^{1} \mathrm{H}$ and ${ }^{13} \mathrm{C}$ NMR studies, and the X-ray diffraction study on $\mathbf{2 b}$.
Table 2. Selected Bond Distances $(\AA)$ and Bond Angles (deg) of $\mathbf{4 a}$ (E sd in Parentheses)

\begin{tabular}{|c|c|c|c|}
\hline $\begin{array}{l}\text { W-Re(1) } \\
\text { W-O(10) } \\
W-C(11) \\
C(10)-C(11) \\
\operatorname{Re}(1)-C(1) \\
\operatorname{Re}(1)-C(3) \\
\operatorname{Re}(2)-C(5) \\
\operatorname{Re}(2)-C(7) \\
\operatorname{Re}(2)-C(9)\end{array}$ & $\begin{array}{l}2.906(1) \\
1.71(1) \\
2.12(2) \\
1.27(2) \\
2.00(2) \\
2.00(2) \\
2.01(2) \\
1.93(2) \\
2.02(2)\end{array}$ & $\begin{array}{l}\operatorname{Re}(1)-\operatorname{Re}(2) \\
W-C(10) \\
\operatorname{Re}(1)-C(10) \\
W-C(1) \\
\operatorname{Re}(1)-C(2) \\
\operatorname{Re}(1)-C(4) \\
\operatorname{Re}(2)-C(6) \\
\operatorname{Re}(2)-C(8)\end{array}$ & $\begin{array}{l}3.010(1) \\
2.12(2) \\
2.01(1) \\
2.41(2) \\
2.05(2) \\
2.02(2) \\
2.02(2) \\
2.00(2)\end{array}$ \\
\hline $\begin{array}{l}\text { W-Re(1)-Re(2) } \\
\operatorname{Re}(1)-\operatorname{Re}(2)-C(7) \\
\operatorname{Re}(1)-C(10)-C(11) \\
\operatorname{Re}-C-O \text { (terminal) }\end{array}$ & $\begin{array}{l}135.64(4) \\
178.1(6) \\
162(1) \\
177(2)\end{array}$ & $\begin{array}{l}\operatorname{Re}(2)-\operatorname{Re}(1)-C(1) \\
\operatorname{Re}(2)-\operatorname{Re}(1)-C(10) \\
\operatorname{Re}(1)-C(1)-O(1)\end{array}$ & $\begin{array}{c}167.5(5) \\
89.0(5) \\
152(2)\end{array}$ \\
\hline
\end{tabular}

Table 3. Selected Bond Distances $(\AA \AA)$ and Bond Angles (deg) of 6a (E sd in Parentheses)

\begin{tabular}{|c|c|c|c|}
\hline $\begin{array}{l}W-\operatorname{Re}(1) \\
\operatorname{Re}(1)-\operatorname{Re}(2) \\
W-C(9) \\
C(9)-C(10) \\
\operatorname{Re}(1)-C(1) \\
\operatorname{Re}(1)-C(3) \\
\operatorname{Re}(2)-C(5) \\
\operatorname{Re}(2)-C(7)\end{array}$ & $\begin{array}{l}3.061(1) \\
3.0943(8) \\
2.057(9) \\
1.39(1) \\
1.95(1) \\
1.941(9) \\
1.96(1) \\
1.97(1)\end{array}$ & $\begin{array}{l}\text { W- } \operatorname{Re}(2) \\
W-O(9) \\
\operatorname{Re}(1)-C(9) \\
\operatorname{Re}(1)-C(10) \\
\operatorname{Re}(1)-C(2) \\
\operatorname{Re}(1)-C(4) \\
\operatorname{Re}(2)-C(6) \\
\operatorname{Re}(2)-C(8)\end{array}$ & $\begin{array}{l}2.7624(8) \\
1.701(6) \\
2.313(8) \\
2.394(8) \\
1.98(1) \\
1.98(1) \\
1.912(9) \\
1.88(1)\end{array}$ \\
\hline $\begin{array}{l}1-\operatorname{Re}(1)-\operatorname{Re}(2) \\
\mathrm{e}(1)-W-\operatorname{Re}(2) \\
(9)-\mathrm{C}(10)-\mathrm{C}(11)\end{array}$ & $\begin{array}{l}53.33(2) \\
63.95(2) \\
124.4(8)\end{array}$ & $\begin{array}{l}\text { W- } \operatorname{Re}(2)-\operatorname{Re}(1) \\
W-C(9)-C(10) \\
\operatorname{Re}-C-O \text { (terminal) }\end{array}$ & $\begin{array}{c}62.72(2) \\
129.0(7) \\
177(1)\end{array}$ \\
\hline
\end{tabular}

As indicated in Figure $\mathbf{1}$, the structure of $\mathbf{2 b}$ consists of an L-shaped core arrangement with eight terminal CO ligands, four on each Re atom. The acetylideligand adopts a novel $\mu_{3}, \eta^{1}, \eta^{1}, \eta^{2}$-mode, which is in sharp contrast to the typical edge-bridging $\mu, \eta^{1}, \eta^{2}$-mode or the face-capping $\mu_{3}, \eta^{1}, \eta^{2}, \eta^{2}$-mode observed in other cluster compounds. ${ }^{10}$ The $\alpha$-carbon $C(9)$ is linked to all three metal atoms with distances in the range 2.160(7)-2.215(9) $\AA$, but the $\beta$-carbon $\mathrm{C}(10)$ is bonded only to the $\mathrm{W}$ atom, with substantial carbenic bonding character,

(10) (a) Cherkas, A. A.; Taylor, N. J .; Carty, A. J . J . Chem. Soc. Chem. Commun. 1990, 385. (b) Deeming, A. J .; Donovan-Mtunzi, S.; Hardcastle, K. J . Chem. Soc., Dalton Trans. 1986, 543. (c) Boyar, E.; Deeming, A. J .; Felix, M. S. B.; Kabir, S. E.; Adatia, T.; Bhusate, R.; McPartlin, M.; Powell, H. R. J . Chem. Soc. Dalton Trans. 1989, 5. 


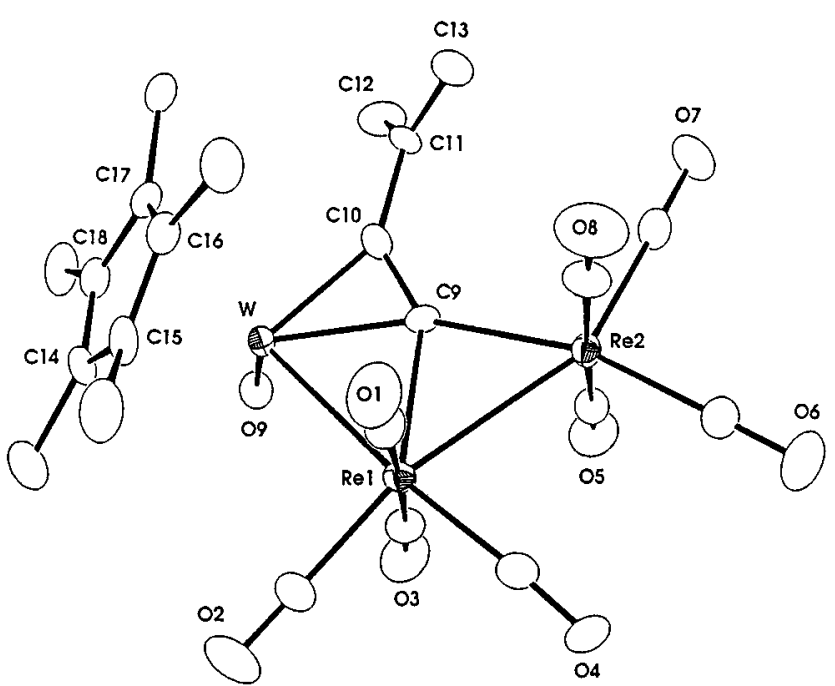

Figure 1. Molecular structure of $\mathrm{Cp} * \mathrm{~W}(\mathrm{O}) \mathrm{Re},[\mathrm{CCC}$ $\left.(\mathrm{Me})=\mathrm{CH}_{2}\right](\mathrm{CO})_{8}(\mathbf{2 b})$ and selected bond lengths $(\AA)$ and angles (deg): $W-\operatorname{Re}(1)=2.7544(8), \operatorname{Re}(1)-\operatorname{Re}(2)=2.9167$ (9), $\mathrm{W}-\mathrm{O}(9)=1.699(6), \mathrm{W}-\mathrm{C}(9)=2.182(9), \mathrm{W}-\mathrm{C}(10)=$ 2.012(9), $\operatorname{Re}(1)-C(9)=2.160(7), \operatorname{Re}(2)-C(9)=2.215(9)$, $C(9)-C(10)=1.31(1), W-C(10)-C(9)=79.0(6), \operatorname{Re}(2)-$ $\mathrm{C}(9)-\mathrm{C}(10)=132.8(7)$.

Table 4. Selected Bond Distances $(\AA \AA)$ and Bond Angles (deg) of 7a (Esd in Parentheses)

\begin{tabular}{|c|c|c|c|}
\hline $\mathrm{W}-\operatorname{Re}(1)$ & $2.850(1)$ & $W-\operatorname{Re}(2)$ & $3.045(1)$ \\
\hline $\operatorname{Re}(1)-\operatorname{Re}(2)$ & $2.983(1)$ & $\mathrm{W}-\mathrm{O}(9)$ & $1.686(9)$ \\
\hline$W-C(9)$ & $2.05(2)$ & $\operatorname{Re}(1)-C(9)$ & $2.20(1)$ \\
\hline $\operatorname{Re}(1)-C(1)$ & $1.92(2)$ & $\operatorname{Re}(1)-C(2)$ & 1.99(1) \\
\hline $\operatorname{Re}(1)-C(3)$ & $1.98(1)$ & $\operatorname{Re}(1)-C(4)$ & 1.93(1) \\
\hline $\operatorname{Re}(2)-C(5)$ & $1.96(2)$ & $\operatorname{Re}(2)-C(6)$ & $1.87(2)$ \\
\hline $\operatorname{Re}(2)-C$ & $1.93(2)$ & $\operatorname{Re}(2)-C(8)$ & $1.76(2)$ \\
\hline$W-\operatorname{Re}(1)-\operatorname{Re}(2)$ & $62.88(2)$ & $W-\operatorname{Re}(2)-\operatorname{Re}(1)$ & $56.43(2)$ \\
\hline $\operatorname{Re}(1)-W-\operatorname{Re}(2)$ & $60.69(3)$ & $\mathrm{W}-\mathrm{C}(9)-\operatorname{Re}(1)$ & $84.3(5)$ \\
\hline$W-\operatorname{Re}(2)-C(5)$ & $78.8(4)$ & $W-\operatorname{Re}(2)-C(7)$ & $102.5(4)$ \\
\hline $\mathrm{W}-\operatorname{Re}(2)-\mathrm{C}(8)$ & $110.4(6)$ & $\mathrm{Re}-\mathrm{C}-\mathrm{O}$ (terminal) & $175(1)$ \\
\hline
\end{tabular}

Table 5. Selected Bond Distances $(\AA \AA)$ and Bond Angles (deg) of 8 (Esd in Parentheses)

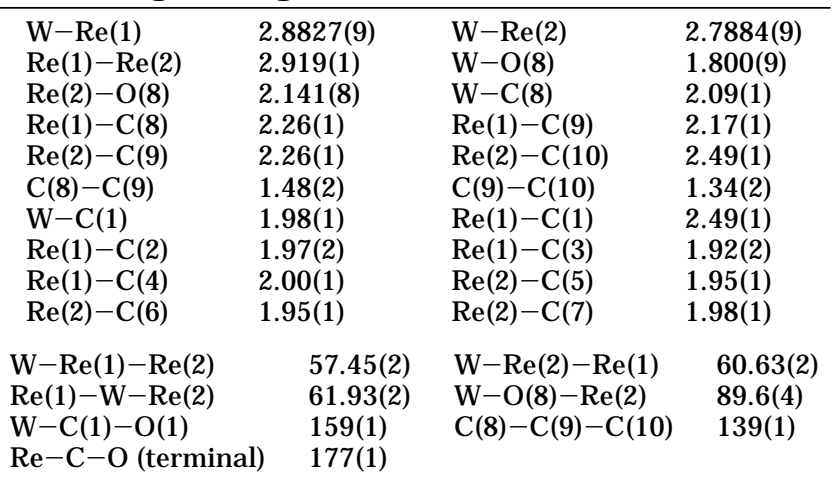

$\mathrm{W}-\mathrm{C}(10)=2.012(9) \AA$. In accordance with this bonding mode, the $\alpha$ - and $\beta$-resonances of the acetylide of $2 \mathbf{a}$ and $\mathbf{2 b}$ appeared at $\delta 184.6$ and $211.4(\mathrm{~J} \mathrm{wc}=77 \mathrm{~Hz})$ and at $\delta 181.9$ and $212.8(\mathrm{~J} \mathrm{wC}=72 \mathrm{~Hz})$ in the ${ }^{13} \mathrm{C} \mathrm{NMR}$ spectrum. The downfield shift of the $\beta$-carbon atoms with respect to the $\alpha$-carbons is obviously due to the $\mathrm{W}=\mathrm{C}$ carbenic character and is confirmed by the observation of large J wc coupling constants.

In addition, the oxo ligand adopts a terminal mode, and the resulting $\mathrm{W}-\mathrm{O}$ vector is perpendicular to the plane defined by the metal atoms and the acetylide ligand. This ligand arrangement is probably due to the availability of the d-orbitals in forming both the $\sigma$-bonding and $\pi$-interaction to the oxygen atom. Although the

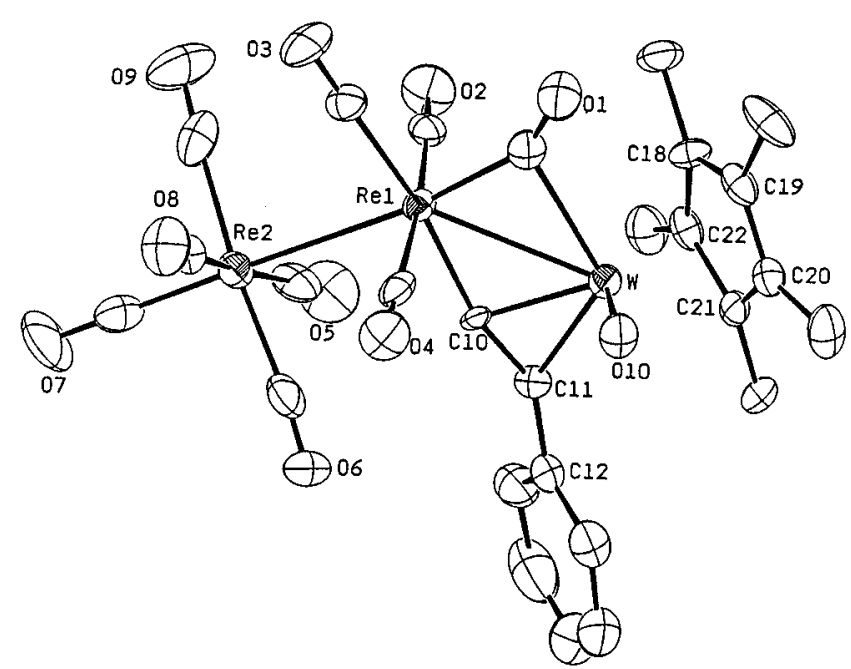

Figure 2. Molecular structure of $\mathrm{Cp} * \mathrm{~W}(\mathrm{O}) \mathrm{Re}_{2}(\mathrm{CCPh})(\mathrm{CO})_{9}$ (4a), showing the atomic labeling scheme and the thermal ellipsoids at $30 \%$ probability level.

$\mathrm{W}-\mathrm{O}(9)$ distance (1.699(6) $\AA$ ) is similar to that observed in $\mathrm{W}_{2} \mathrm{Fe}$ and $\mathrm{WCO}$ oxo compounds ${ }^{11}$ and in the $\mathrm{W}_{2} \mathrm{Ru}_{4}$ cluster with the $\mathrm{Cp} * \mathrm{~W}(\mathrm{O})$ fragment, 12 it cannot be utilized for identifying the nature of bonding, as the actual bonding mode (double bond vs triple bond) is determined by its donor capability (two-electron vs fourelectron on a covalent model) and because the respective $\mathrm{W}-\mathrm{O}$ interactions show little variation in bond distances. ${ }^{13}$ However, we propose that the $\mathrm{W}-\mathrm{O}$ interaction presents a $\mathrm{W} \equiv \mathrm{O}$ triple bond on the basis of electron counting. In this way, if the oxo ligand is regarded as a four-electron donor in the coval ent model, the number of valence el ectrons satisfies the prediction of 50 electrons for a trinuclear compound with two metal-metal bonds.

Conversely, the relatively short $\mathrm{W}-\mathrm{Re}(1)$ distance $(2.7544(8) \AA$ ) observed in $\mathbf{2} \mathbf{b}$ falls between that of the $\mathrm{W}=\mathrm{Re}$ double bond $(2.672 \AA)$ and $\mathrm{W}$ - Re single bond $(2.898 \AA)$ in the WRe $e_{2}$ cluster $\mathrm{Cp}^{*} \mathrm{WRe}_{2}(\mu-\mathrm{H})[\mathrm{CHCHC}$ $(\mathrm{Me}) \mathrm{CH}](\mathrm{CO})_{7}{ }^{14}$ This suggests that this $\mathrm{W}-\mathrm{Re}(1)$ bonding may possess some $\mathrm{W}=$ Re double-bond bonding character. This leads us to believe that the actual bonding interaction of this cluster contains some contribution from the second structure, shown. The latter

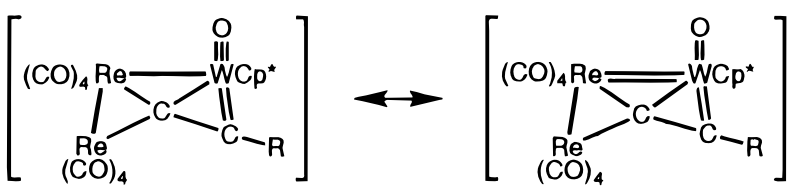

possesses $\mathrm{W}=\mathrm{Re}$ and $\mathrm{W}=\mathrm{O}$ double bonds and is complementary to the originally proposed structure involving a $\mathrm{W}-\mathrm{Re}$ single bond and a $\mathrm{W} \equiv \mathrm{O}$ triple bond.

Complexes $\mathbf{2 a}$ and $\mathbf{2} \mathbf{b}$ were transformed into two new compounds $\mathrm{Cp} * \mathrm{~W}(\mathrm{O}) \mathrm{Re}_{2}(\mathrm{CCPh})(\mathrm{CO})_{9}(\mathbf{4 a}, \mathrm{R}=\mathrm{Ph} ; \mathbf{4} \mathbf{b}$, $\mathrm{R}=\mathrm{C}(\mathrm{Me})=\mathrm{CH}_{2}$ ) in nearly quantitative yields, when heated to $90{ }^{\circ} \mathrm{C}$ under a $\mathrm{CO}$ atmosphere in toluene. An ORTEP drawing of derivative $\mathbf{4 a}$ is shown in Figure 2 ,

(11) (a) Busetto, L.; J effery, J . C.; Mills, R. M.; Stone, F. G. A.; Went M. J ; Woodward, P. J. Chem. Soc., Dalton Trans. 1983, 101. (b) EI Amin, A. E.; J effery, J. C.; Walters, T. M. J . Chem. Soc., Chem. Commun. 1990, 170.

(12) Su, C.-J :; Su, P.-C.: Chi, Y.; Peng, S.-M.; Lee, G.-H. J . Am Chem. Soc. 1996, 118, 3289.

(13) Mayer, J. M. Inorg. Chem. 1988, 27, 3899.

(14) Cheng, P.-S.; Chi, Y.; Peng, S.-M.; Lee, G.-H. Organometallics 1993, 12, 250. 
and selected distances and angles are given in Table 1. The molecule was found to consist of an open triangular geometry with five terminal $\mathrm{CO}$ ligands attached to the $\operatorname{Re}(2)$ atom and three terminal and one semi-bridging $\mathrm{CO}$ ligand to the $\mathrm{Re}(1)$ atom. The equatorial $\mathrm{CO}$ ligands on $\mathrm{Re}$ atoms adopt the staggered conformation, as expected on steric grounds. The $\operatorname{Re}(1)-\operatorname{Re}(2)$ distance of 3.010(1) $\AA$ is similar to that of $\operatorname{Re}_{2}(C O)_{10}$ (3.041(1) $\AA)^{15}$ and the isonitrile compounds $\operatorname{Re}_{2}(C O)_{x} L_{10-x}, x=$ $1-3(3.048-3.081 \AA) .{ }^{16}$ The $W-\operatorname{Re}(1)-\operatorname{Re}(2)$ angle is $135.64(4)^{\circ}$, which is more obtuse than that observed in its precursor $\mathbf{2 b}\left(99.88(2)^{\circ}\right)$ but is comparable to that reported in the open trinuclear clusters $\mathrm{Os}_{3}(\mathrm{CO})_{11}(\mu$ $\left.\mathrm{CNCF}_{3}\right)_{2}\left(140.39(4)^{\circ}\right)$ and $\mathrm{Os}_{3}\left(u-\mathrm{CNCF}_{3}\right)_{2}(\mathrm{CO})_{10}(\mathrm{NCMe})$ $\left(137.06(2)^{\circ}\right)$, which contain at least one bridging isonitrile ligand on the Os-Os bond. ${ }^{17}$

The acetylide ligand in $\mathbf{4 a}$ is associated with the $\mathrm{Re}-\mathrm{W}$ bond. The $\mathrm{C}(10)-\mathrm{C}(11)$ distance $(1.27(2) \AA)$ is slightly longer than those of the terminal acetylide ligands $(1.19-1.21 \AA)^{18}$ and is al so comparable with that of the dinuclear oxo-acetylide complex $\mathrm{Cp} * \operatorname{Re}(\mathrm{CO})$ $(\mathrm{PMe} 2 \mathrm{Ph})\left(\mu-\mathrm{C}_{2} \mathrm{Ph}\right) \mathrm{W}(\mathrm{O}) \mathrm{Cp}(1.30(2) \AA),{ }^{19}$ indicating the existence of a weakened donor interaction from the acetylide ligand to the $\mathrm{W}$ atom. On the basis of this structural data and the elongated $\mathrm{W}-\mathrm{C}(10)$ and $\mathrm{W}-\mathrm{C}(11)$ distances observed, the acetylide is considered to behave as a three-el ectron donor, forming $\sigma$-bond to the central $\operatorname{Re}(1)$ atom and $\pi$-interaction to the $\mathrm{W}$ atom, respectively. In accordance, the ${ }^{13} \mathrm{C}$ NMR signals of the acetylide $\alpha$ - and $\beta$-carbon atoms occur at $\delta 175.6$ and 176.3 and at $\delta 147.6$ and 148.8 with coupling constant $\mathrm{J} w c=60-61 \mathrm{~Hz}$ for complexes $\mathbf{4 a}$ and $\mathbf{4 b}$, respectively. These high-field shifts with respect to those observed in $\mathbf{2}$ are consistent with the reduction of electron donation to the $W$ atom via an interaction incorporating one set of $\pi$-orbitals. ${ }^{20}$

Reaction of the Oxo-Acetylide Complex 2a with Hydrogen. Three new products were obtained from the hydrogenation of $\mathbf{2 a}$ in heptane solution (1 atm, 90 $\left.{ }^{\circ} \mathrm{C}, 40 \mathrm{~min}\right)$. These compounds were identified as $\mathrm{Cp} * \mathrm{WRe}_{2}(\mu-\mathrm{O})(u-\mathrm{H})_{2}(\mathrm{CCPh})(\mathrm{CO})_{6}(\mathbf{5 a}$, dark-red, 50\%), $\mathrm{Cp} * \mathrm{~W}(\mathrm{O}) \mathrm{Re}_{2}(\mu-\mathrm{CHCHPh})(\mathrm{CO})_{8}(6 \mathbf{a}$, orange, $28 \%)$, and Cp*W(O) $\operatorname{Re}_{2}(\mu-\mathrm{H})\left(\mu-\mathrm{CHCH}_{2} \mathrm{Ph}\right)(\mathrm{CO})_{8}(7 \mathrm{a}$, yellow, 5\%) and were characterized by spectroscopic methods and $\mathrm{X}$-ray diffraction studies.

For complex $\mathbf{5 a}$, the ${ }^{1} \mathrm{H}$ NMR spectrum exhibits two signals at $\delta-7.44(\mathrm{~J} \mathrm{wH}=135 \mathrm{~Hz})$ and -14.08 in addition to the $\mathrm{Cp}^{*}$ and the phenyl signals, indicating the presence of two bridging hydride ligands. The acetylide signals appear at $\delta 156.6$ and $121.6(\mathrm{Jc}=$ $71 \mathrm{~Hz}$ ) in the ${ }^{13} \mathrm{C}$ NMR spectrum, which fall in the range expected for the acetylides with $\mu_{3}-\eta^{2}$-mode. ${ }^{21}$ As can

(15) Churchill, M. R.; Amoh, K. N.; Wasserman, H. J . Inorg. Chem. 1981, 20, 1609.

(16) Harris, G. W.; Boeyens, J . C. A.; Coville, N.J . Organometallics 1985, 4, 914.

(17) (a) Adams, R. D.; Chi, Y.; DesMarteau, D. D.; Lentz, D.; Marschall, R. J . Am. Chem. Soc. 1992, 114, 1919. (b) Adams, R. D.; Chi, Y.; DesMarteau, D. D.; Lentz, D.; Marschall, R.; Scherrmann, A. J . Am. Chem. Soc. 1992, 114, 10822.

(18) Manna, J .; J ohn, K. D.; Hopkins, M. D. Adv. Organomet. Chem. 1995, 38, 79.

(19) Lai, N.-S.; Tu, W.-C.; Chi, Y.; Peng, S.-M.; Lee, G.-H. Organometallics 1994, 13, 4652.

(20) (a) Templeton, J . L.; Ward, B. C. J . Am. Chem. Soc. 1980, 102, 3288. (b) Templeton, J . L. Adv. Organomet. Chem. 1989, $29,1$.

(21) (a) Hwang, D.-K.; Chi, Y.; Peng, S.-M.; Lee, G.-H. Organome tallics 1990, 9, 2709. (b) Carty, A. J .; Cherkas, A. A.; Randall, L. H. Polyhedron 1988, 7, 1045. (c) Chi, Y.; Su, P.-C.; Peng, S.-M.; Lee, G.H. Organometallics 1995, 14, 5483.

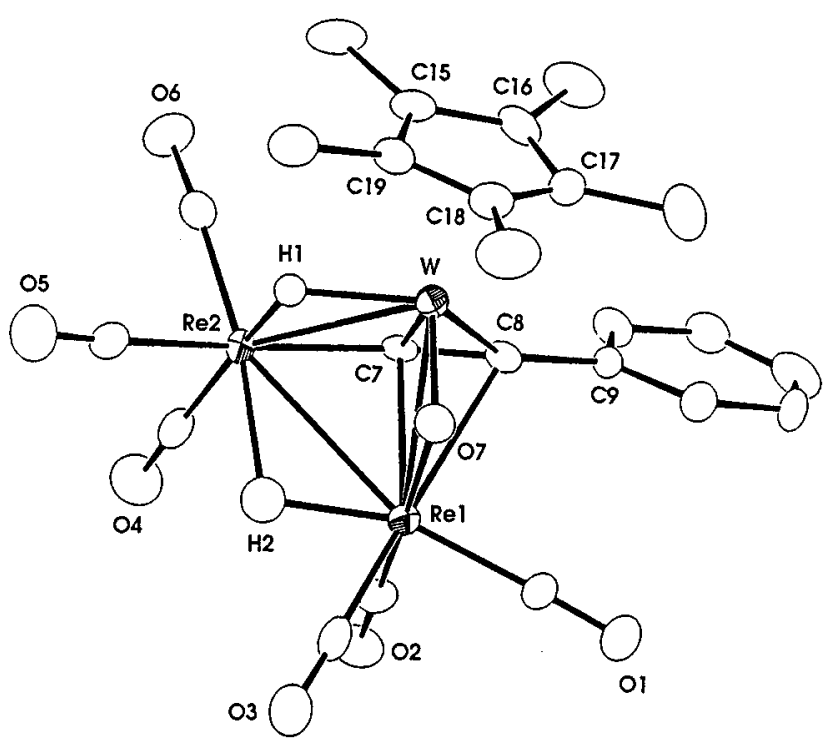

Figure 3. Molecular structure of $C p * W R e_{2}(\mu-O)(\mu-\mathrm{H})_{2-}$ $(\mathrm{CCPh})(\mathrm{CO})_{6}(\mathbf{5 a})$ and selected bond lengths $(\AA)$ and angles (deg): $\operatorname{Re}(1)-\operatorname{Re}(2)=3.0412(5), \operatorname{Re}(1)-W=2.8652(6), \operatorname{Re}-$ $(2)-\mathrm{W}=2.9918(6), \mathrm{W}-\mathrm{O}(7)=1.765(6), \operatorname{Re}(1)-\mathrm{O}(7)=$ 2.286(5), $\mathrm{W}-\mathrm{C}(7)=2.189(8), \operatorname{Re}(1)-\mathrm{C}(7)=2.370(8)$, Re$(2)-\mathrm{C}(7)=2.028(7), \mathrm{W}-\mathrm{C}(8)=2.118(8), \mathrm{Re}(1)-\mathrm{C}(8)=$ $2.496(8), \mathrm{C}(7)-\mathrm{C}(8)=1.34(1), \mathrm{W}-\mathrm{H}(1)=1.78(6), \mathrm{Re}(2)-$ $\mathrm{H}(1)=1.86(6), \mathrm{Re}(1)-\mathrm{H}(2)=1.78(7), \mathrm{Re}(2)-\mathrm{H}(2)=2.03-$ (8), $\mathrm{W}-\mathrm{O}(7)-\mathrm{Re}(1)=89.1(2), \operatorname{Re}(2)-\mathrm{C}(7)-\mathrm{C}(8)=157.2(7)$, $\mathrm{C}(7)-\mathrm{C}(8)-\mathrm{C}(9)=138.7(8)$.

be seen from Figure 3, the X-ray diffraction analysis reveals the triangular $\mathrm{WRe}_{2}$ core geometry. The hydride ligands, which are unambiguously located on the electron density map, span the $\mathrm{W}-\operatorname{Re}(2)$ and the $\mathrm{Re}$ (1) $-\operatorname{Re}(2)$ edge. Each Re atom is coordinated by three terminal CO ligands, and the acetylide is perpendicular to the $\mathrm{W}-\mathrm{Re}(1)$ edge supporting the bridging oxo ligand. The oxo ligand has changed to an asymmetric $\mathrm{W}=\mathrm{O} \rightarrow \mathrm{Re}$ bridging mode with distances $\mathrm{W}-\mathrm{O}(7)=1.765(6) \AA$ and $\operatorname{Re}(1)-\mathrm{O}(7)=2.286(5) \AA$. These distances are similar to those in the $\mathrm{WRu}_{4}$ cluster $\mathrm{Cp} * \mathrm{~W}(\mathrm{O})_{2} \mathrm{Ru}_{4}\left(\mu_{4}-\mathrm{PPh}\right)$ $(\mathrm{CCPh})(\mathrm{CO})_{10} 22$ and the trinuclear cluster $\mathrm{Cp}_{2} \mathrm{~W}_{2} \mathrm{Re}(u$ $\mathrm{Br})(\mu-\mathrm{O})(\mu-\mathrm{CTOl})_{2}(\mathrm{CO})_{3}{ }^{23}$ and are also related to the $\mathrm{W}=\mathrm{O} \rightarrow \mathrm{O}$ s mode of cluster compounds having the $\mathrm{WO}_{3}-$ $(\mu-\mathrm{O})$ core arrangement. ${ }^{24}$

Compound $\mathbf{6 a}$ is a trinuclear oxo-alkenyl complex with the same number of $\mathrm{CO}$ ligands. As depicted in Figure 4, the cluster core contains one $\mathrm{Cp} * \mathrm{~W}(\mathrm{O})$ fragment and two $\operatorname{Re}(\mathrm{CO})_{4}$ units, with the alkenyl ligand $\mu-\mathrm{CH}=\mathrm{CHPh}$ bridging the longer $\mathrm{W}-\mathrm{Re}(1)$ bond. Both Re atoms appear to adopt a pseudo-octahedral arrangement. The trans-alkenyl ligand, which is formally generated by the addition of $\mathrm{H}_{2}$ across the acetylide, adopts the typical $\sigma+\pi$ bonding mode. Consistent with this assignment were the ${ }^{1} \mathrm{H}$ NMR data of two doublets at $\delta 5.93$ and 4.72 with coupling constant ${ }^{3} \mathrm{~J} \mathrm{HH}=13.6$ $\mathrm{Hz}$ and the distinctive signals at $\delta 161.3 \mathrm{~N} \mathrm{wC}=115$ $\mathrm{Hz}$ ) and 76.8 in its ${ }^{13} \mathrm{C}$ NMR spectrum.

The alkylidene complex $\mathbf{7 a}$ is the third product generated from this reaction. It can be obtained independently in high yield from hydrogenation of the

(22) Blenkiron, P.; Carty, A. J .; Peng, S.-M.; Lee, G.-H.; Su, C.-J .; Shiu, C.-W.; Chi, Y. Organometallics 1997, 16, 519.

(23) Carriedo, G. A.; J effery, J . C.; Stone, F. G. A. J . Chem. Soc., Dalton Trans. 1984, 1597.

(24) (a) Chi, Y.; Shapley, J. R.; Ziller, J. W.; Churchill, M. R. Organometallics 1987, 6, 301. (b) Gong, J.-H.; Chen, C.-C.; Chi, Y.; Wang, S.-L.; Liao, F.-L. J . Chem. Soc., Dalton Trans. 1993, 1829. 


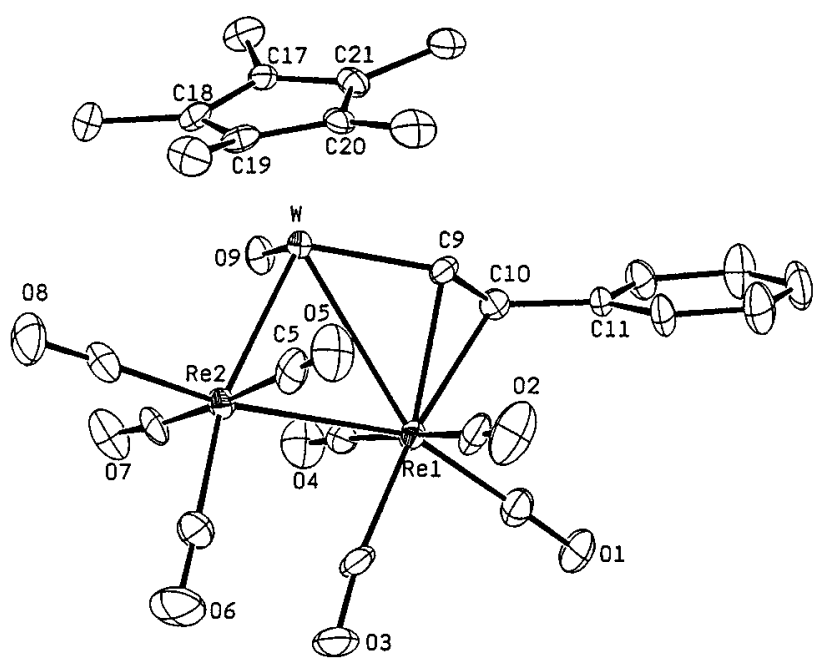

Figure 4. Molecular structure of $\mathrm{Cp}^{*} \mathrm{~W}(\mathrm{O}) \mathrm{Re}_{2}(\mathrm{CHCHPh})-$ $(\mathrm{CO})_{8}(\mathbf{6 a})$, showing the atomic labeling scheme and the thermal ellipsoids at $30 \%$ probability level.

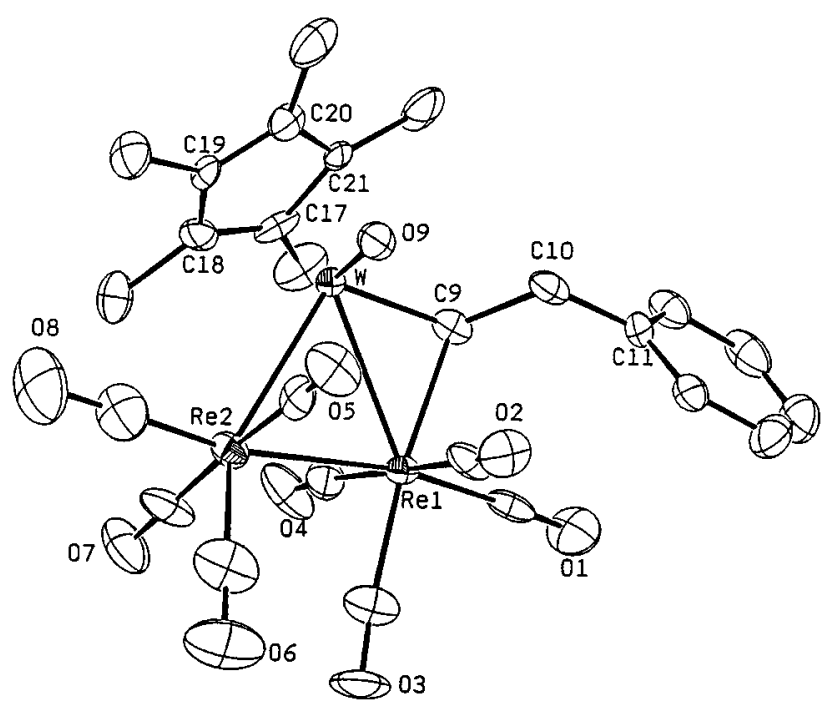

Figure 5. Molecular structure of $\mathrm{Cp} * \mathrm{~W}(\mathrm{O}) \mathrm{Re}_{2}(u-\mathrm{H})\left(\mathrm{CHCH}_{2-}\right.$ $\mathrm{Ph})(\mathrm{CO})_{8}(\mathbf{7 a})$, showing the atomic labeling scheme and the thermal ellipsoids at $30 \%$ probability level.

alkenyl complex $\mathbf{6} \mathbf{a}$ in toluene. The key spectral data comprises the ${ }^{13} \mathrm{C}$ NMR signals at $\delta 141.6(\mathrm{~J} \mathrm{wc}=104$ $\mathrm{Hz}$ ) and 61.7 due to the $\alpha$-carbon of the alkylidene and the adjacent methylene group. The ${ }^{1} \mathrm{H}$ NMR spectrum agrees with this assignment, exhibiting three signals at $\delta 7.15,4.65$, and 4.13 with an ABX splitting pattern and a hydride signal at $\delta-10.93(\mathrm{JH}=97.2 \mathrm{~Hz}$ ). The ORTEP diagram depicted in Figure 5 exhibits the expected triangular $\mathrm{WRe}_{2}$ skel etal arrangement with the alkylidene ligand, $\mu-\mathrm{CHCH}_{2} \mathrm{Ph}$, residing on the $\mathrm{W}-\operatorname{Re}(1)$ bond (2.950(1) $\AA$ ). The hydride ligand, which is not located by difference Fourier synthesis, spans the second $\mathrm{W}-\mathrm{Re}$ bond because of its longer bond distance (3.045(1) A). The enlarged W-Re(2)-C(7)O angle (102.5$\left.(4)^{\circ}\right)$, which deviates substantially from the $90^{\circ}$ angle of the octahedral environment, further confirms the assignment of hydride. Finally, the $\alpha$-hydrogen of the alkylidene ligand is tilting away from the $\mathrm{W} \equiv \mathrm{O}$ fragment. This orientation reflects the absol ute configuration of the alkenyl ligand in its precursor $\mathbf{6 a}$.

Reaction of the Oxo-Vinylacetylide Complex $\mathbf{2 b}$ with Hydrogen. Treatment of the vinylacetylide complex $\mathbf{1 b}$ with hydrogen in refluxing toluene (1 atm, $30 \mathrm{~min}$ ) afforded four products. These compounds were
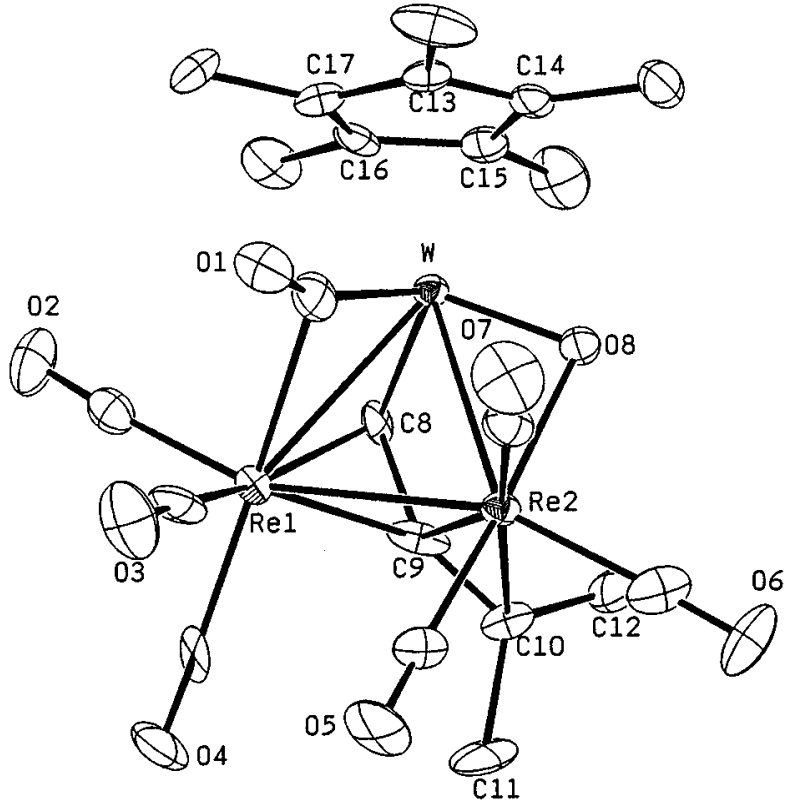

Figure 6. Molecular structure of $\mathrm{Cp}^{*} \mathrm{WRe}_{2}(u-\mathrm{O})(\mathrm{CHC}-$ $\left.\mathrm{CMe}_{2}\right)(\mathrm{CO})_{7}(\mathbf{8})$, showing the atomic labeling scheme and the thermal ellipsoids at $30 \%$ probability level.

identified as acetylide complex Cp*WRe $(\mu-\mathrm{O})(u-\mathrm{H})_{2}[\mu-$ $\left.\mathrm{CCC}(\mathrm{Me})=\mathrm{CH}_{2}\right](\mathrm{CO})_{6}(\mathbf{5 b}, 32 \%)$, alkenyl complex Cp*W(O) $\operatorname{Re}_{2}\left[\mu-\mathrm{CHCHC}(\mathrm{Me})=\mathrm{CH}_{2}\right](\mathrm{CO})_{8}(\mathbf{6 b}, 15 \%)$, alkylidene complex Cp*W(O)Re $(\mu-\mathrm{H})\left[\mu-\mathrm{CHCH}_{2} \mathrm{C}(\mathrm{Me})=\mathrm{CH}_{2}\right](\mathrm{CO})_{8}$ (7b, 4\%), and allenyl complex Cp*WRe $(\mu-\mathrm{O})[\mu-\mathrm{CHCC}-$ $\left.(\mathrm{Me})_{2}\right](\mathrm{CO})_{7}(\mathbf{8}, 8 \%)$. The first three compounds were identified according to their IR and ${ }^{1} \mathrm{H}$ and ${ }^{13} \mathrm{C} N M R$ data, as their structures were analogous to the phenyl complexes $\mathbf{5 a}, \mathbf{6 a}$, and $\mathbf{7 a}$ mentioned in the preceding section. The cluster $\mathbf{8}$ corresponds an unknown al lenyl cluster, and its structure was further established crystallographically.

As indicated by the ORTEP diagram (Figure 6), the molecule consists of a WRe triangular geometry in which each $\mathrm{Re}$ atom is coordinated by three terminal $\mathrm{CO}$ ligands and the seventh $\mathrm{CO}$ ligand is bridging across a $\mathrm{W}-\mathrm{Re}$ bond. The bridging oxo ligand spans the $\mathrm{W}-\mathrm{Re}(2)$ bond and falls on the extension of the $\mathrm{WRe}_{2}$ plane. The allenyl ligand adopts a normal $\mu_{3}-\eta^{1}, \eta^{2}, \eta^{2}$ mode ${ }^{25}$ with the $\mathrm{C}_{\alpha}-\mathrm{C}_{\beta}$ vector parallel to the $\mathrm{W}-\mathrm{Re}(1)$ edge. The $\mathrm{C}_{3}$ backbone of this allenyl ligand is bent, with the $\mathrm{C}(8)-\mathrm{C}(9)-\mathrm{C}(10)$ angle of $139(1)^{\circ}$ being comparable to those reported $\left(138(2)-152(1)^{\circ}\right)$ for the trinuclear allenyl complexes. ${ }^{26}$ The $\mathrm{M}-\mathrm{C}_{\gamma}$ distance (Re$(2)-C(10)=2.49(1) \AA)$ is significantly longer than the other $\mathrm{M}-\mathrm{C}_{\alpha}$ and $\mathrm{M}-\mathrm{C}_{\beta}$ bonds within the molecule (2.09(1) $-2.26(1) \AA)$. Such a large deviation in $M-C$ distances suggests the existence of a weakened bonding interaction between the Re atom and the $\mathrm{C}_{\beta}-\mathrm{C}_{\gamma}$ fragment. The short $C(9)-C(10)$ distance of $1.34(2) \AA$, which is close to that of the $\mathrm{C}=\mathrm{C}$ double bond in free ethylene (1.34 $\AA$ ), conforms with this observation. This elongation of the $\mathrm{M}-\mathrm{C} \gamma$ bond in the allenyl cluster $\mathbf{8}$ has been seen in the related allenylidene cluster $\mathrm{Cp}^{*} \mathrm{WRe}_{2}(\mu$-OMe)$\left[\mathrm{CCC}(\mathrm{Me})_{2}\right](\mathrm{CO})_{8}{ }^{5}$ but has not been reported for the

(25) (a) Wojcicki, A.; Shuchart, C. E. Coord. Chem. Rev. 1990, 105, 35. (b) Wojcicki, A. J. Cluster Sci. 1993, 4, 59.

(26) (a) Shuchart, C. E.: Wojcicki, A.; Calligaris, M.; Faleschini, P. Nardin, G. Organometallics 1994, 13, 1999. (b) Nucciarone, D.; MacL aughlin, S. A.; Taylor, N.J .; Carty, A. J . Organometallics 1988, 7, 106. (c) Suades, J .; Dahan, F.; Mathieu, R. Organometallics 1988 7, 47. (d) Gervasio, G.; Osella, D.; Valle, M. Inorg. Chem. 1976, 5, 1221. 
Scheme 2

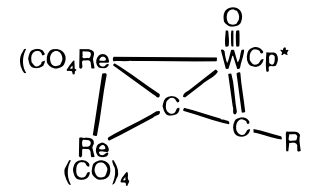

(2) $\mathrm{R}=\mathrm{Ph}, \mathrm{C}(\mathrm{Me})=\mathrm{CH}_{2}$

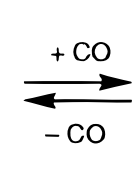

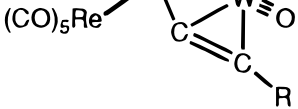

(3) $\mathrm{R}=\mathrm{Ph}, \mathrm{C}(\mathrm{Me})=\mathrm{CH}_{2}$ allenyl cluster. ${ }^{27}$ Finally, the ${ }^{13} \mathrm{C}$ NMR signals of the allenyl ligand appears at $\delta 165.0\left(\mathrm{C}_{\beta}\right), 136.0 \mathrm{~J} \mathrm{wc}=97$ $\left.\mathrm{Hz}, \mathrm{C}_{\alpha}\right)$, and $66.0\left(\mathrm{C}_{\gamma}\right)$, which fall in the chemical shift range expected for the related allenyl clusters.

\section{Discussion}

Oxidation of the acetylide complexes $\mathbf{1}$ with oxygen produced the corresponding oxo cluster compounds $\mathbf{2}$. After the elimination of a $\mathrm{CO}$ ligand as $\mathrm{CO}_{2}$, the framework opens up to give an L-shaped geometry. The possible reaction stoichiometry is shown in eq 1 : In

$$
\begin{aligned}
\mathrm{Cp} * W \mathrm{Re}_{2}(\mathrm{C} \equiv \mathrm{CR})(\mathrm{CO})_{9}+\mathrm{O}_{2} \rightarrow & \\
& \mathrm{Cp} * \mathrm{~W}(\mathrm{O}) \mathrm{Re}_{2}(\mathrm{C} \equiv \mathrm{CR})(\mathrm{CO})_{8}+\mathrm{CO}_{2}
\end{aligned}
$$

addition, replacing oxygen with other mild oxidant, e.g, nitrous oxide, increases the yiel ds by $5-10 \%$. Substantial decomposition was noted when the reaction was carried out in refluxing toluene at $110^{\circ} \mathrm{C}$. The successful isolation of $\mathbf{2}$ in high yields demonstrates the potential of using oxygen or nitrous oxide as a source in generation of metal complexes and cluster compounds containing oxo ligands. ${ }^{28}$ This method complements the various oxidative decarbonylation strategies using iodosylbenzene, ${ }^{29}$ hydrogen peroxide, ${ }^{30}$ dioxirane, ${ }^{31}$ and air oxidation. ${ }^{32}$

The acetylide ligand in $\mathbf{2}$ adopts a novel $\mu_{3}-\eta^{1}, \eta^{1}, \eta^{2}-$ mode with $\mathrm{C}_{\alpha}$ connected to all three metal atoms and $\mathrm{C}_{\beta}$ coordinated to a $\mathrm{W}$ atom via a double bond. No such bonding mode has ever been reported in the system of acetylide clusters. ${ }^{33}$ We believe that this mode has some connection with the el ectron deficiency of the $\mathrm{Cp} * \mathrm{~W}(\mathrm{O})$ fragment, induced by the coordination of the electronegative oxo ligand.

Upon heating under $\mathrm{CO}$ atmosphere, complexes 2 absorb one $\mathrm{CO}$ ligand, forming the clusters $\mathbf{4}$ with one $\operatorname{Re}(\mathrm{CO})_{4}$ unit converting to the $\operatorname{Re}(\mathrm{CO})_{5}$ unit and with the acetylide ligand changing to the edge-bridging mode (Scheme 2). As a result, the terminal $\operatorname{Re}(\mathrm{CO})_{5}$ unit is no longer connected to the acetylide ligand. The free rotation around the $\mathrm{Re}-\mathrm{Re}$ bond becomes attainable under this circumstance. The observation of a broad ${ }^{13} \mathrm{C}$ NMR signal at $\delta \mathbf{1 9 0 . 9}$ for $\mathbf{4 a}$ and $\delta 191.0$ for $\mathbf{4 b}$ at room temperature with a ratio corresponding to four $\mathrm{CO}$ ligands, which are assigned to the equatorial CO ligands

(27) (a) Young, G. H.; Raphael, M. V.; Wojcicki, A.; Calligaris, M.; Nardin, G.; Bresciani-Pahor, N. Organometallics 1991, 10, 1934. (b) Doherty, S.; Corrigan, J . F.; Carty, A. J .; Sappa, E. Adv. Organomet. Chem. 1995, 37, 39.

(28) Bottomley, F.; Chen, J. Organometallics 1992, 11, 3404.

(29) Kim, J.-H.; Hong, E.; Kim, J .; Do, Y. Inorg. Chem. 1996, 35, 5112.

(30) (a) Bottomley, F.; Boyle, P. D.; Chen, J . Organometallics 1994, 13, 370. (b) Thiel, W. R.; Fischer, R. W.; Herrmann, W. A. J. Organomet. Chem. 1993, 459, C9.

(31) Bolowiec, S.; Kochi, J. K. Inorg. Chem. 1991, 30, 1215.

(32) Alt, H. G.; Hayen, H. I.; Rodgers, R. D. J . Chem. Soc., Chem. Commun. 1987, 1795.

(33) (a) Sappa, E.; Tiripicchio, A.; Braunstein, P. Chem. Rev. 1983, 83, 203. (b) Sappa, E. J . Cluster Sci. 1994, 5, 211. (c) J eannin, Y. Transition Met. Chem. (London) 1993, 18, 122.

\section{Scheme 3}

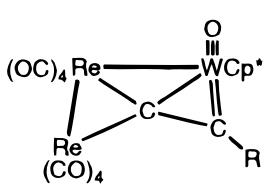

(2a) $\mathrm{R}=\mathrm{Ph}$
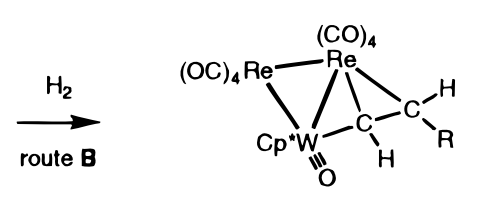

(6a) $\mathrm{R}=\mathrm{Ph}$

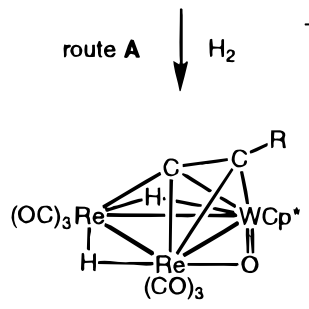

(5a) $\mathrm{R}=\mathrm{Ph}$<smiles>CCCCCCCC</smiles>

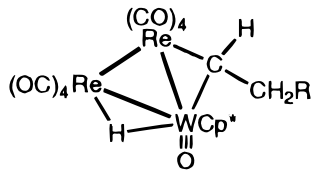

(7a) $\mathrm{A}=\mathrm{Ph}$

on the terminal $\operatorname{Re}(\mathrm{CO})_{5}$ unit, support the existence of such dynamic motion in solution. Pyrolysis of complexes 4 in the absence of $\mathrm{CO}$ leads to the regeneration of the corresponding complexes $\mathbf{2}$ in high yields. These experiments suggest that the interconversions between $\mathbf{2}$ and $\mathbf{4}$ are fully reversible.

In contrast to the simplicity of $\mathrm{CO}$ addition, hydrogenation of $\mathbf{2 a}$ afforded a mixture of three compounds $\mathbf{5 a}, \mathbf{6 a}$, and $7 \mathbf{a}$ (Scheme 3). Upon increasing the temperature to $110{ }^{\circ} \mathrm{C}$ in toluene, the yield of $\mathbf{5 a}$ dropped substantially while the total yields for $\mathbf{6} \mathbf{a}$ and 7a increased only moderately. These experiments suggest that $\mathbf{5 a}$ is unstable while $\mathbf{6} \mathbf{a}$ and $\mathbf{7 a}$ are thermodynamically more favorable.

In principle, the transformation from $\mathbf{2}$ to 5, 6, and $\mathbf{7}$ can be understood within the framework of two competing pathways. The key step for the first pathway (route A of Scheme 2) involves the removal of at least one CO prior to the initial coordination of $\mathrm{H}_{2}$ or immediately after the addition of $\mathrm{H}_{2}$. The formation of $\mathbf{5}$ requires the loss of a second CO ligand, which leads to the formation of the triangular metal framework. Interestingly, when $\mathbf{5 a}$ was treated with $\mathrm{CO}$ in refluxing dichloromethane solution, it slowly gave $\mathbf{6 a}$ in low yield, together with a small amount of $\mathbf{2 a}$ by loss of a $\mathrm{H}_{2}$ molecule. This reactivity pattern reveals the delicate balance of the dehydrogenation reaction vs the hydride migration to the acetylide ligand.

On the other hand, the formation of alkenyl complexes 6 and alkylidene complexes 7 involves the direct addition of $\mathrm{H}_{2}$ without the prior elimination of $\mathrm{CO}$ ligand (route B). This postulation is supported by the fact that complexes 2, 6, and $\mathbf{7}$ own the same number of $\mathrm{CO}$ ligands. It is possible that $\mathrm{H}_{2}$ first coordinates to the $W \mathrm{We}_{2}$ framework of $\mathbf{2}$ to afford a dihydride intermediate with a hypothetical formula Cp*W(O) $\operatorname{Re}_{2}(u-\mathrm{H})_{2}(\mathrm{CCR})$ $(\mathrm{CO})_{8}$, which then converts to the alkenyl complexes 6 by transferring both hydrides to the acetylide. The prospect involving the direct addition of $\mathrm{H}_{2}$ over the acetylide is eliminated because of the trans configuration observed for the alkenyl ligand.

Complexes 6 then react further with another $\mathrm{H}_{2}$ molecule to form the alkylidene ligand in 7. The addition of the second $\mathrm{H}_{2}$ molecule is highly regioselective because of the isolation of only one alkylidene isomer. This proposed sequential addition of $\mathrm{H}_{2}$ is further supported by the $D_{2}$ labeling experiments. Treatment of $\mathbf{6 a}$ or $\mathbf{6 b}$ with $\mathrm{D}_{2}$ afforded the labeled 


\section{Scheme 4}

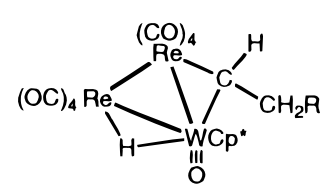

(7b) $\mathrm{R}=\mathrm{C}(\mathrm{Me})=\mathrm{CH}_{2}$
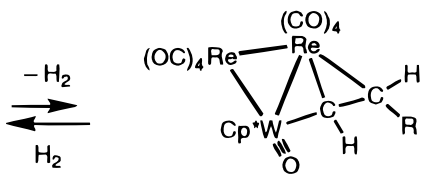

(6b) $\mathrm{R}=\mathrm{C}(\mathrm{Me})=\mathrm{CH}_{2}$
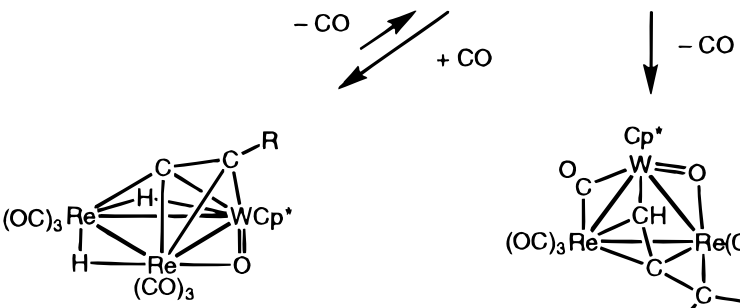

(5b) $\mathrm{R}=\mathrm{C}(\mathrm{Me})=\mathrm{CH}_{2}$

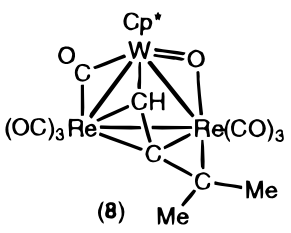

complexes 7, Cp*W(O)Re $2(\mu-\mathrm{D})(\mu-\mathrm{CHCHDR})(\mathrm{CO})_{8}(\mathrm{R}=$ $\mathrm{Ph}$ or $\left.\mathrm{C}(\mathrm{Me})=\mathrm{CH}_{2}\right)$ in which the deuterium atoms selectively replaced the hydride and one methylene hydrogen of the alkylidene ligand.

The unsaturation required for the activation of $\mathrm{H}_{2}$ on the $\mathrm{WRe}_{2} \mathrm{O}$ core in both $\mathbf{2}$ and $\mathbf{6}$ may be generated through (i) a reversible cleavage of the metal-metal bond or (ii) a reversible two-electron reduction in donor capability of the oxo ligand, as indicated in eq 2 . The

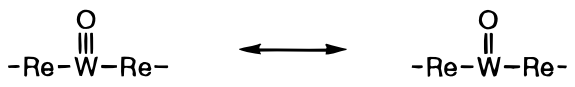

reversible conversion between complexes $\mathbf{2}$ and $\mathbf{4}$ is a precedent to process $i$. Process $i i$ has been proposed to occur, in an attempt to account for the greater affinity of the oxo-alkylidyne cluster $\mathrm{CpWO}_{3}(\mu-\mathrm{O})\left(\mu_{3}-\mathrm{CH}_{2} \mathrm{Tol}\right)$ $(\mathrm{CO})_{9}$ with various reagents including $\mathrm{CO}, \mathrm{H}_{2}$, phosphines, alkynes, and alkenes. ${ }^{34}$ We cannot eliminate either possibility based on our present experimental data. In addition, thermolysis of $\mathbf{7 a}$ or $\mathbf{6 a}$ under $\mathrm{N}_{2}$ generates a mixture of $\mathbf{7 a}, \mathbf{6} \mathbf{a}$, and $\mathbf{5 a}$ or a mixture of $\mathbf{6 a}, \mathbf{5 a}$, and $\mathbf{2 a}$, respectively. These experiments clearly demonstrate that the dehydrogenation sequence, a reverse transformation from $\mathbf{7}$ to $\mathbf{6}$ and then a mixture of $\mathbf{2}$ and $\mathbf{5}$, is feasible and involves the same activating process.

For the hydrogenation of the vinylacetylide complex $\mathbf{2 b}$, in addition to the expected mixture of $\mathbf{5 b}, \mathbf{6 b}$, and $\mathbf{7 b}$, we observed the formation of the allenyl cluster compound $\mathbf{8}$ in low yield (Scheme 4). The relationship between compounds 5, 6, 7, and $\mathbf{8}$ was established on the basis of the reaction stoichiometry and their reactivities. According to our previous delineation, two possible reaction pathways can be proposed to account for the production of $\mathbf{8}$. The first one involves a concerted addition of two hydrogen atoms to the $\mathrm{C}_{\alpha}$

(34) (a) Chi, Y.; Shapley, J . R.; Churchill, M. R.; Fettinger, J J C. J . Organomet. Chem. 1989, 372, 273. (b) Park, J . T.; Chi, Y.; Shapley, J. R.; Churchill, M. R.; Ziller, J. W. Organometallics 1994, 13, 813. (c) Churchill, M. R.; Bueno, C.; Park, J . T.; Shapley, J . R. Inorg. Chem. 1984, 23, 1017. position and the remote methylene group of the vinylacetylide ligand $\mathrm{C} \equiv \mathrm{CC}(\mathrm{Me})=\mathrm{CH}_{2}$ in $\mathbf{2 b}$. Alternatively, a concerted or a transition metal mediated $1,3-\mathrm{H}$ migration from the $\mathrm{C}_{\beta}$ position of the $\mathrm{CH}=\mathrm{CHC}$ $(\mathrm{Me})=\mathrm{CH}_{2}$ ligand in $\mathbf{6 b}$ to the methylene site, along with the elimination of $\mathrm{CO}$, would give the same product $\mathbf{8}$. We favor the second mechanism, as thermolysis of both al kenyl complex $\mathbf{6 b}$ and al kylidene complex $\mathbf{7 b}$ have all afforded the allenyl complex $\mathbf{8}$ in moderate yields. Finally, this transformation from vinylalkenyl to allenyl via the 1,3-H-migration is related conceptually to the conversion of an alkyne (HCCR) to a vinylidene (CCHR) on trinuclear clusters via a 1,2-H-shift. ${ }^{35}$

\section{Conclusion}

In summary, we present here the facile synthesis of $W \mathrm{We}_{2}$ clusters containing the oxo ligand and the subsequent conversion of the ligated acetylide to alkenyl, alkylidene, and allenyl upon treatment with hydrogen and the thermally induced isomerization. The oxo ligand in these complexes adopts either the terminal or the asymmetrical, doubly-bridging mode. The precise role of the oxo ligand in the conversions between $\mathbf{2} \leftrightarrow \mathbf{4}$ and $\mathbf{2} \rightarrow \mathbf{6} \rightarrow \mathbf{7}$ remains in doubt, although we have demonstrated that this reaction pattern is suppressed in the absence of an oxo ligand. Thus, the parent complex 1 failed to react with $\mathrm{CO}$. Carbonylation of the hydrido-acetylide cluster $\mathrm{Cp}^{*} \mathrm{WRe}_{2}(\mu-\mathrm{H})_{2}(\mathrm{CCPh})(\mathrm{CO})_{8}$ (9), ${ }^{5}$ which is related to $5 \mathbf{a}$ by formally replacing the bridging oxo ligand with two CO ligands, produced only elimination of $\mathrm{H}_{2}$. Whether the contribution of the oxo

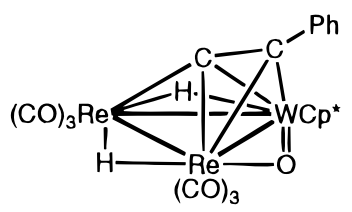

(5a)

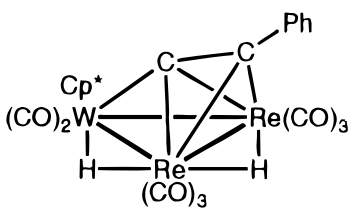

(9) ligand via its high electronegativity and its capability to shuttle between a four- and six-el ectron donor ${ }^{33,36}$ and to adopt various bonding modes are the key factors to promote such reactions awaits the results of continuing explorations.

Acknowledgment. We thank the National Science Council of the Republic of China for financial support (Grant No. NSC 85-2113-M007-008).

Supporting Information Available: Tables of atomic coordinates and anisotropic thermal parameters for complexes $\mathbf{4 a}, \mathbf{6 a}, \mathbf{7 a}$, and 8 (16 pages). Ordering information is given on any current masthead page.

\section{OM 9609426}

(35) (a) Cooksey, C. J .; Deeming, A. J .; Rothwell, I P. J . Chem. Soc. Dalton Trans. 1981, 1718. (b) Vahrenkamp, H.; Roland, E. J . Mol Catal. 1983, 21, 233. (c) Bantel, H.; Powell, A. K.; Vahrenkamp, H. Chem. Ber. 1990, 123, 1607.

(36) (a) Shapley, J . R.; Park, J . T.; Churchill, M. R.; Ziller, J . W.; Beanan, L. R. J . Am. Chem. Soc. 1984, 106, 1144. (b) Chi, Y.; H wang, L.-S.; Lee, G.-H.; Peng, S.-M. J . Chem. Soc., Chem. Commun. 1988, 1456. 